\title{
Physics of the Ion Composition Boundary: a comparative 3-D hybrid simulation study of Mars and Titan
}

\author{
S. Simon ${ }^{1}$, A. Boesswetter ${ }^{1}$, T. Bagdonat ${ }^{1}$, and U. Motschmann ${ }^{1,2}$ \\ ${ }^{1}$ Institute for Theoretical Physics, TU Braunschweig, Germany \\ ${ }^{2}$ Institute for Planetary Research, DLR, Berlin, Germany
}

Received: 3 May 2006 - Revised: 4 December 2006 - Accepted: 6 December 2006 - Published: 1 February 2007

\begin{abstract}
The plasma environments of Mars and Titan have been studied by means of a 3-D hybrid simulation code, treating the electrons as a massless, charge-neutralizing fluid, whereas ion dynamics are covered by a kinetic approach. As neither Mars nor Titan possesses a significant intrinsic magnetic field, the upstream plasma flow interacts directly with the planetary ionosphere. The characteristic features of the interaction region are determined as a function of the alfvénic, sonic and magnetosonic Mach number of the impinging plasma. For the Martian interaction with the solar wind as well as for the case of Titan being located outside Saturn's magnetosphere in times of high solar wind dynamic pressure, all three Mach numbers are larger than 1. In such a scenario, the interaction gives rise to a so-called Ion Composition Boundary, separating the ionospheric plasma from the ambient flow and being highly asymmetric with respect to the direction of the convective electric field. The formation of these features is explained by analyzing the Lorentz forces acting on ionospheric and ambient plasma particles. Titan's plasma environment is highly variable and allows various different combinations of the three Mach numbers. Therefore, the Ion Composition Boundary may vanish under certain circumstances. The relevant physical mechanism is illustrated as a function of the Mach numbers in the upstream plasma flow.
\end{abstract}

Keywords. Magnetospheric physics (Magnetosphere interactions with satellites and rings; Magnetosphere-ionosphere interactions; Planetary magnetospheres)

\section{Introduction}

Since neither Mars nor Titan possesses a significant intrinsic magnetic field, the ionospheres of these planets are di-

Correspondence to: S. Simon

(sven.simon@tu-bs.de) rectly exposed to the ambient plasma flow (Sauer et al., 1990; Riedler et al., 1991; Acuña et al., 1998; Lundin et al., 2004; Ness et al., 1982; Neubauer et al., 1984; Backes et al., 2005). In the case of Mars, the planetary ionosphere interacts directly with the solar wind, whose alfvénic, sonic and magnetosonic Mach number are always larger than 1 . The ionosphere of Titan might be exposed to the Saturnian magnetospheric plasma as well as to the solar wind. Due to Titan's plasma environment being highly variable, a variety of different combinations of Mach numbers in the upstream flow can be studied. The Mach numbers of the upstream plasma flow have a decisive character for the major features of the interaction region. On the one hand, these numbers determine whether a bow shock is formed in front of the obstacle. If a shock is formed, the deflection of the impinging flow around the obstacle is significantly stronger than in a scenario where such a boundary is missing. On the other hand, the simulations presented in this paper will also illustrate that the Mach numbers of the upstream plasma control the degree to which the upstream flow is capable of mixing with the cold ionospheric plasma population.

In the case of Mars, the interaction with the solar wind gives rise to a set of sharply pronounced plasma boundaries in the vicinity of the obstacle. Due to the supersonic nature of the impinging solar wind, a bow shock is formed in front of the obstacle. The interaction also leads to the formation of a so-called Ion Composition Boundary, separating the solar wind ions from the cold plasma of ionospheric origin. Ion measurements conducted by the ASPERA and HARP experiments aboard Phobos-2 identified an Ion Composition Boundary (ICB) which was located at the same position as the magnetic pile-up boundary (MPB). This boundary separates the solar wind protons from the planetary ions (Sauer et al., 1990; Breus et al., 1991; Sauer et al., 1994). The magnetic pile-up boundary at Mars has been observed between the bow shock and the ionopause by both Mars Global Surveyor and the Phobos-2 MAGMA instrument (Riedler et al.,

Published by Copernicus GmbH on behalf of the European Geosciences Union. 
1991; Vignes et al., 2000). A detailed discussion of the Martian plasma boundaries, especially of the MPB, has been given by Nagy et al. (2004) who summarize the data obtained by MGS and Phobos 2. Barabash and Lundin (2006) give an overview of the instruments aboard ASPERA 3/ MarsExpress and present first scientific results concerning the plasma boundaries. Upon crossing the boundary, the magnetic field vector rotates, its mean amplitude begins to increase, fluctuations are reduced, and superthermal electron fluxes begin to decrease. The same structures have been observed at active comets like Halley and Grigg-Skjellerup by the Giotto mission (Mazelle et al., 1989; Rème et al., 1993). The plasma environment of Venus also features a strong analogy to the Martian situation. An extensive study of the plasma boundaries at Mars and Venus has been conducted by Bertucci et al. (2005b). In contrast to the terminology used in this paper, Lundin et al. (2004) called this boundary induced magnetosphere boundary (IMB). They used this definition of the IMB due to the lack of magnetic field instruments aboard Mars Express, avoiding conflicts with defined features such as the magnetic pile-up boundary (Vignes et al., 2000). Based on their analysis of MGS magnetic field data, Bertucci et al. (2004) identify a significant magnetic field enhancement near the MPB. It is confirmed (Bertucci et al., 2005a) that the MPB is a well-defined plasma boundary which can be characterized as a tangential discontinuity.

Recently, several hybrid simulation studies (cf. Shimazu, 2001; Terada et al., 2002; Kallio and Janhunen, 2002; Bößwetter et al., 2004; Modolo et al., 2005) have focussed on the Martian plasma environment. These simulations clearly indicate that the structure of the Martian plasma environment, especially the magnetic pile-up region at the obstacle's dayside, possess a pronounced asymmetry with respect to the direction of the convective electric field. In this paper, it will be demonstrated that global 3-D hybrid simulations of the Martian plasma environment enable to obtain a kinetic explanation for the formation of the Ion Composition Boundary. The mechanism being essential for the Ion Composition Boundary will be discussed by analyzing the forces acting on solar wind ions as well as on particles of ionospheric origin. In the Martian scenario, the alfvénic Mach number in the undisturbed solar wind is of the order of $M_{A} \approx 10$. In general, the Martian scenario will help us understand the physics of the Ion Composition Boundary in the case of all three Mach numbers in the upstream flow being significantly larger than 1. In contrast to this, in the Titan scenario, all three Mach numbers are of the order of 1 . Specifically, transitions between regions with different Mach numbers are a major characteristic of Titan's plasma environment.

Titan possesses an extended neutral atmosphere and orbits Saturn in a distance of 20.3 Saturn radii and an orbital period of 15.95 days. Titan's orbit is located in the outer regions of Saturn's magnetosphere for average solar wind conditions. Due to Titan's orbital period being considerably larger than Saturn's rotational period, Titan is permanently exposed to a flow of magnetized plasma with a relative velocity around $120 \mathrm{~km} / \mathrm{s}$. Because Titan does not possess a significant intrinsic magnetic field, the upstream plasma flow interacts directly with the ionosphere in a similar way as the ionosphere of Mars interacts with the solar wind. However, the Titan scenario possesses several unique features. On the one hand, Titan's dayside ionosphere is not necessarily located in the hemisphere that is exposed to the upstream plasma flow. On the other hand, the upstream plasma flow in Saturn's outer magnetosphere is super-alfvénic, yet subsonic and submagnetosonic. A special situation occurs when Titan is located in Saturn's magnetotail as in this region the upstream plasma flow is subalfvénic, subsonic and submagnetosonic (Schardt et al., 1984; Wolf and Neubauer, 1982). A third scenario can be realized when Saturn's magnetosphere is compressed due to high solar wind dynamic pressure. In this case, Titan might even be able to leave Saturn's magnetosphere in the subsolar region of its orbit and interact directly with the solar wind. In the case of Titan being exposed to the solar wind, it will be shown that an Ion Composition Boundary is formed in analogy to the Martian situation: the solar wind is separated from the ionospheric plasma flow, the structure of the interaction region is symmetric in a plane perpendicular to the convective electric field and exhibits a pronounced asymmetry with respect to the direction of $\boldsymbol{E}_{\text {conv }}=-\boldsymbol{u}_{i} \times \boldsymbol{B}$ (Brecht et al., 2000; Simon et al., 2006b). Cassini measurements (Wahlund et al., 2005) as well as multiple numerical simulation studies (Ledvina and Cravens, 1998; Kabin et al., 1999; Brecht et al., 2000; Kallio et al., 2004; Ledvina et al., 2004; Simon et al., 2006b) indicate that when Titan is located inside Saturn's magnetosphere, the Ion Composition Boundary is no longer existent. In this paper, we investigate the mechanism leading to the disappearence of the Ion Composition Boundary by presenting a set of 3-D hybrid simulations: In our first simulation run, the alfvénic Mach number $M_{A}$, the sonic Mach number $M_{S}$ and the magnetosonic Mach number $M_{M S}$ of the upstream plasma flow are all larger than 1. A second simulation scenario $\left(M_{A}>1, M_{S}>1, M_{M S}<1\right)$, although it does not occur in reality, will help understand the Ion Composition Boundary's transition to the case of Titan being located inside the outer regions of Saturn's magnetosphere. The other runs of the sequence refer to the cases of Titan being located in the Saturnian outer magnetosphere and in the magnetotail. However, in contrast to earlier simulations (Simon et al., 2006b), this scenario does not show the situation at the equinoxes of Saturn's orbit, i.e. Titan is not shielded from the solar UV radiation by Saturn. As the Cassini flybys of Titan occur in late Saturnian south summer (Backes et al., 2005; Backes, 2005), this case may be closer to the measured Titan situation than the case of an obstacle that is entirely protected from the solar UV radiation by Saturn. The paper concludes with a summary, illustrating the transition of the Ion Composition Boundary's characteristic features when altering the upstream Mach numbers from high to low values. Although serveral comparisons between 
Titan and the unmagnetized planets Venus and Mars are already available (Verigin et al., 1984; Luhmann et al., 1991), neither of them focusses on the structure of the Ion Composition Boundary.

\section{Simulation model}

The simulations are carried out by using a 3-D hybrid code that can operate on an arbitrary curvilinear grid (Bagdonat and Motschmann, 2001, 2002a,b; Bagdonat, 2004). The present version of the code has already been successfully applied to the solar wind interaction with magnetized asteroids (Simon et al., 2006a) as well as to the plasma environments of Mars (Bößwetter et al., 2004) and Titan (Simon et al., 2006b). Since an extensive discussion of the major features of the simulation code is given by Bößwetter et al. (2004) and Simon et al. (2006b), only a short overview of the most important aspects will be presented in the following paragraphs.

In the hybrid model, the electrons are treated as a massless fluid, whereas all ion species occuring in the simulation are described as individual particles. The basic equations of this model can be written as follows:

- Equations of motion for individual ions:

$$
\frac{\mathrm{d} \boldsymbol{x}_{s}}{\mathrm{~d} t}=\boldsymbol{v}_{s} \quad \text { and } \quad \frac{\mathrm{d} \boldsymbol{v}_{s}}{\mathrm{~d} t}=\frac{q_{s}}{m_{s}}\left\{\boldsymbol{E}+\boldsymbol{v}_{s} \times \boldsymbol{B}\right\},
$$

where $\boldsymbol{x}_{s}$ and $\boldsymbol{v}_{s}$ denote the position and the velocity of an ion of species $s$, respectively. The vectors $\boldsymbol{E}$ and $\boldsymbol{B}$ are the electromagnetic fields. The ion mass and velocity are denoted by $m_{s}$ and $q_{s}$, respectively.

- Electric field equation:

$$
\boldsymbol{E}=-\boldsymbol{u}_{i} \times \boldsymbol{B}+\frac{(\nabla \times \boldsymbol{B}) \times \boldsymbol{B}}{\mu_{0} e n_{e}}-\frac{\nabla P_{e, 1}+\nabla P_{e, 2}}{e n_{e}}
$$

where $\boldsymbol{u}_{i}$ is the mean ion velocity. The plasma is quasineutral, i.e. the mean ion $\left(n_{i}\right)$ and electron density $\left(n_{e}\right)$ are assumed to be equal. As in general, the electron temperature in a planetary ionosphere differes significantly from the electron temperature in the ambient plasma flow, two different electron pressure terms $P_{e, 1}$ and $P_{e, 2}$ have been incorporated into the simulation model. Both electron populations are described by adiabatic laws:

$$
P_{e, 1} \propto \beta_{e, 1} n_{e, 1}^{\kappa} \quad \text { and } \quad P_{e, 2} \propto \beta_{e, 2} n_{e, 2}^{\kappa},
$$

where $\kappa$ is the adiabatic exponent. For the simulations presented in this work, a value of $\kappa=2$ has been chosen (Bößwetter et al., 2004; Simon et al., 2006a,b).

- Magnetic field equation: by using Faraday's law, an expression describing the time evolution of the magnetic field can be obtained:

$$
\frac{\partial \boldsymbol{B}}{\partial t}=\nabla \times\left(\boldsymbol{u}_{i} \times \boldsymbol{B}\right)-\nabla \times\left[\frac{(\nabla \times \boldsymbol{B}) \times \boldsymbol{B}}{\mu_{0} e n_{e}}\right] .
$$

Because of the adiabatic description of the electrons, the electron pressure terms do not occur in this equation:

$$
\begin{aligned}
& \nabla \times\left(\frac{\nabla n_{e}^{\kappa}}{n_{e}}\right) \\
& =\frac{1}{n_{e}} \nabla \times\left[\nabla\left(n_{e}^{\kappa}\right)\right]+\left[\nabla \frac{1}{n_{e}}\right] \times\left[\nabla\left(n_{e}^{\kappa}\right)\right] \\
& =\left[\nabla \frac{1}{n_{e}}\right] \times\left[\nabla\left(n_{e}^{\kappa}\right)\right] \\
& =-\frac{1}{n_{e}^{2}}\left[\nabla n_{e}\right] \times \kappa n_{e}^{\kappa-1}\left[\nabla n_{e}\right] \\
& =0 .
\end{aligned}
$$

The simulations are carried out on a curvilinear simulation grid (a so-called Fisheye Grid) which can be adapted to the spherical geometry of the obstacle. It also allows an extremely high spatial resolution in the immediate vicinity of the planet. This gird is obtained from an equidistant Cartesian grid by means of a non-linear coordinate transformation. In both the Mars and the Titan simulations, the maximum resolution achieved by using such a grid is well below 0.05 planetary radii, even though this resolution is maintained for only a few cells. The number of macroparticles placed in each cell at the beginning of the simulation is of the order of 8-12.

The obstacle's dayside ionosphere is described by a Chapman layer, i.e. the local ion production rate $q(r, \chi)$ depends on both the altitude $r$ above the surface and the solar zenith angle $\chi$. The nightside production profile is assumed to be independent of the solar zenith angle and is set to an altitudedepending value of $q=q\left(r, \chi=87^{\circ}\right)$ for the Martian as well as for the Titan scenario. In the case of Mars, the ionosphere is assumed to be consisting of atomic oxygen, whereas in the case of Titan, molecular nitrogen ions are the only species that is taken into account by the simulation model. Any ion hitting the so-called Inner Boundary is removed from the simulation. In the case of Mars, this boundary coincides with the surface of the obstacle at a distance of one Mars radius from the center of the simulation domain, whereas it is located at the lower boundary of the ionosphere at an altitude of $1000 \mathrm{~km}$ above the surface in the Titan scenario. This is consistent with earlier hybrid simulations carried out by Kallio et al. (2004). No boundary conditions are imposed on the electromagnetic fields, i.e. the equations for $\boldsymbol{E}$ and $\boldsymbol{B}$ are solved outside as well as inside the obstacle. Although this set of boundary conditions allows the magnetic field to diffuse into the obstacle, it has proven to be an extremely good approximation in earlier studies of the plasma environment of planetary obstacles (Bößwetter et al., 2004; Simon et al., 2006b). 


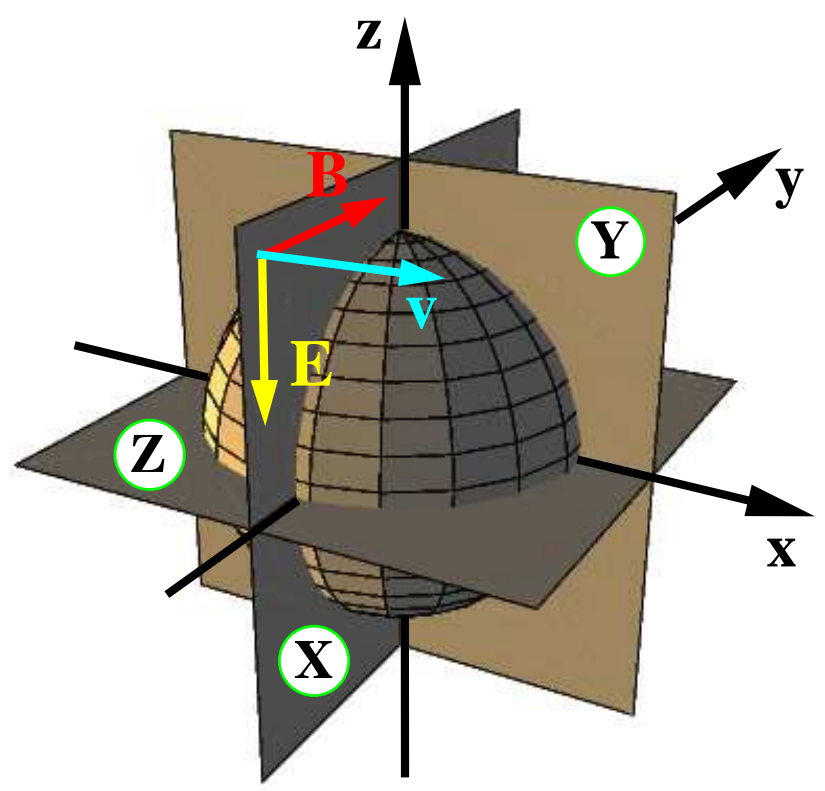

Fig. 1. Plasma environment of Mars - Simulation geometry. The undisturbed plasma flow is directed along the $(+x)$-axis, whereas the homogeneous solar wind magnetic field is parallel to the (+y)-axis. Hence, the convective electric field, defining the direction of asymmetry, is oriented antiparallel to the z-axis. The obstacle's dayside is located in the $(x<0)$ hemisphere. In the figure, the cutting planes $(x=0),(y 0)$ and $(z 0)$ are denoted by the abbreviations $\mathrm{X}, \mathrm{Y}$ and $\mathrm{Z}$, respectively.

\section{The Martian plasma environment $\left(M_{A}>1, M_{S}>1\right.$,} $M_{M S}>1$ )

In order to illustrate the Martian interaction with the solar wind, a 3-D hybrid simulation has been carried out. The simulation geometry is displayed in Fig. 1: the undisturbed solar wind density and magnetic field are given by $n_{S W, 0}=4 \mathrm{~cm}^{-3}$ and $\boldsymbol{B}_{S W, 0}=(0,3 \mathrm{nT}, 0)$, respectively. The upstream plasma flow is directed parallel to the $(+x)$-axis, the upstream alfvénic Mach number is set to $M_{A}=10$. As the electron and proton temperatures in the undisturbed solar wind have been set to $T_{e}=2 \times 10^{5} \mathrm{~K}$ and $T_{p}=5 \times 10^{4} \mathrm{~K}$, the corresponding plasma betas in the undisturbed flow are $\beta_{e}=3.1$ and $\beta_{i}=0.8$. This yields values of

$M_{S}=\frac{M_{A}}{\sqrt{\frac{\kappa}{2}\left(\beta_{e}+\beta_{i}\right)}}=5.1$

and

$M_{M S}=\frac{M_{A}}{\sqrt{\frac{\kappa}{2}\left(\beta_{e}+\beta_{i}\right)+1}}=4.5$

for the sonic and the magnetosonic Mach number, respectively.

Figure 2 displays the simulation results for the polar plane, coinciding with the $(x, z)$ plane of the coordinate system. It can be seen in Figs. 2a and $b$ that downstream of the bow shock, the decelerated solar wind is clearly separated from the ionospheric plasma flow by means of an Ion Composition Boundary, i.e. the solar wind density is high in regions where the ionospheric density is low and vice versa. It is obvious that the structure of the ionospheric tail as well as the magnetic pile-up region at the Martian dayside exhibit a pronounced asymmetry with respect to the direction of the convective electric field.

In the following, we will try to understand the mechanism giving rise to the Ion Composition Boundary by means of a kinetic approach, i.e. by considering the Lorentz forces acting on individual ions of solar wind and ionospheric origin. These forces are given by

$\boldsymbol{F}_{S W}=e\left(\boldsymbol{E}+\boldsymbol{v}_{S W} \times \boldsymbol{B}\right)$

for a solar wind particle and by

$\boldsymbol{F}_{H I}=e\left(\boldsymbol{E}+\boldsymbol{v}_{H I} \times \boldsymbol{B}\right)$

for a heavy ion of ionospheric origin. For the formation of the Ion Composition Boundary, two different contributions to the electric field are of major importance: On the one hand, this is the convective electric field term

$\boldsymbol{E}_{c}=-\boldsymbol{u}_{i} \times \boldsymbol{B}$.

By introducing the average densities $n_{S W}$ and $n_{H I}$ of the solar wind and the heavy ion component, this term can be written as

$$
\begin{aligned}
& \boldsymbol{E}_{c}= \\
& =-\left(\frac{n_{S W}}{n_{S W}+n_{H I}} \boldsymbol{u}_{S W}+\frac{n_{H I}}{n_{S W}+n_{H I}} \boldsymbol{u}_{H I}\right) \times \boldsymbol{B},
\end{aligned}
$$

where $\boldsymbol{u}_{S W}$ and $\boldsymbol{u}_{H I}$ denote the bulk velocities of the solar wind and the heavy ion component, respectively. As can be seen from Eq. (11), the convective electric field is dominated by the fast solar wind velocity in regions where the ionospheric ion density is small, whereas in regions of high heavy ion density, the slow velocity $\boldsymbol{u}_{H I}$ gives the major contribution to the convective electric field.

On the other hand, the electron pressure terms are of important consequence for the formation of the Ion Composition Boundary:

$\boldsymbol{E}_{\nabla}=-\frac{\nabla P_{e, S W}+\nabla P_{e, H I}}{e\left(n_{e, S W}+n_{e, H I}\right)}$.

The electrons are assumed to be adiabatic with an adiabatic exponent of $\kappa=2$. In regions where one of the two ion species is predominant, the electric field arising from the electron pressure term depends only on the density gradient of the respective species, but not on the absolute value of the density. 

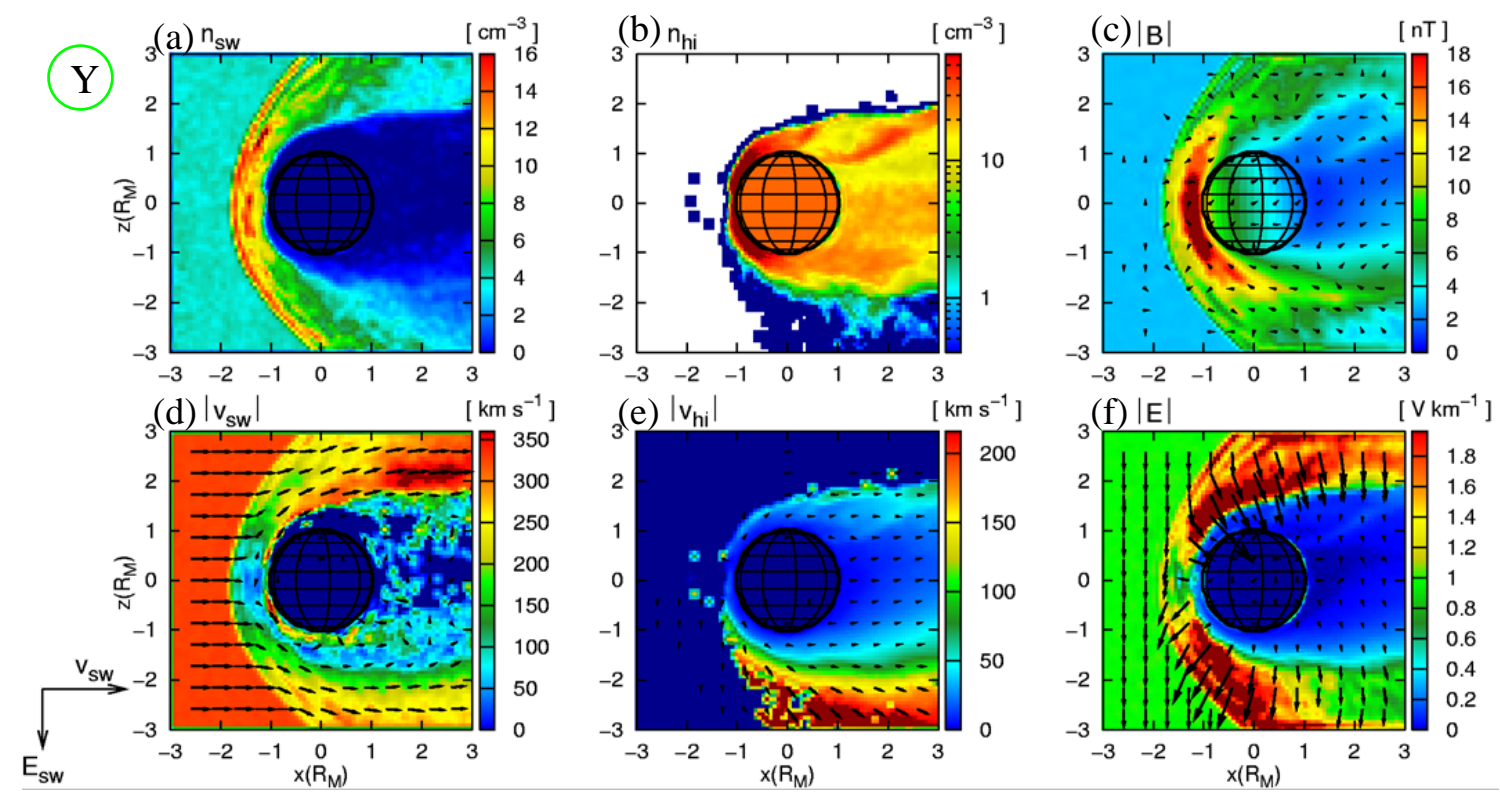

Fig. 2. Plasma environment of Mars - Results of a 3-D hybrid simulation. The figure shows a cut through the polar plane. The quantities displayed in the figure are (a) the solar wind density, (b) the ionospheric heavy ion density, (c) the magnetic field, (d) the solar wind velocity, (e) the heavy ion velocity and (f) the electric field. In the Martian scenario the individual particle velocities $\boldsymbol{v}_{S W}$ in the upstream flow do not differ significantly from the mean plasma velocities $\boldsymbol{u}_{S W}$. Therefore, the arrow $v_{S W}$ on the left side of the figure denotes both a representative particle velocity and the mean plasma velocity. Mach numbers of the upstream plasma flow: $M_{A}=10.0$ (alfvénic), $M_{S}=5.1$ (sonic) and $M_{M S}=4.5$ (magnetosonic).

For instance, in regions where the solar wind particles are predominant, one obtains

$$
\begin{aligned}
& \boldsymbol{E}_{\nabla} \approx-\frac{\nabla P_{e, S W}}{e n_{e, S W}}= \\
& =-\frac{\kappa\left(n_{e, S W}\right)^{\kappa-1} \nabla n_{e, S W}}{e n_{e, S W}}=-\frac{2 \nabla n_{e, S W}}{e} .
\end{aligned}
$$

It is interesting to notice that this term depends only on the density gradient $\nabla n_{e, S W}$, but not on the absolute density value $n_{e, S W}$.

Outside the ionospheric tail region, the solar wind ions are the predominant species. For this reason, the convective electric field outside the tail can be written as

$\boldsymbol{E}_{c} \approx-\boldsymbol{u}_{S W} \times \boldsymbol{B}$.

Now we consider the situation near the flank of the ionospheric tail in the northern hemisphere of the polar plane, referring to a heavy ion that is about to enter the protondominated plasma flow from inside the tail region. The heavy ion trys to cross the tail's northern boundary from inward to outward. As the individual velocity $\boldsymbol{v}_{H I}$ of such a heavy ion is significantly smaller than the flow velocity of the solar wind, the Lorentz force acting on the particle is given by

$$
\begin{aligned}
& \boldsymbol{F}_{H I}=e\left[\left(\boldsymbol{v}_{H I}-\boldsymbol{u}_{S W}\right) \times \boldsymbol{B}-\frac{2 \nabla n_{e, S W}}{e}\right] \\
& \approx e\left[-\boldsymbol{u}_{S W} \times \boldsymbol{B}-\frac{2 \nabla n_{e, S W}}{e}\right] .
\end{aligned}
$$

Due to the relatively high velocity of the shocked solar wind in this region (cf. Fig. 2d) and the magnetic field enhancement in the lobes (cf. Yeroshenko et al., 1990; Bößwetter et al., 2004), the first term including the magnetic field is the predominat one, i.e.

$\boldsymbol{F}_{H I} \approx-e \boldsymbol{u}_{S W} \times \boldsymbol{B}$.

The solar wind flow is directed almost parallel to the $(+x)$ direction, whereas the magnetic field is almost perpendicular to the polar plane. Therefore, the force acting on the heavy ion is directed perpendicular to the tail and points toward the tail region. This force is the major reason for the tail to be forbidden to expand into the northern hemisphere. The direction of this force is determined by the orientation of the convective electric field at the tail's northern flank. This is consistent with Fig. 2f, showing that at the tail's upper flank, the electric field is directed perpendicular to the tail flank and points inward. Due to the ionospheric plasma velocity in the tail region being significantly smaller than the undisturbed solar wind velocity (cf. Figs. $2 \mathrm{~d}$ and $2 \mathrm{e}$ ), the convective electric field almost vanishes inside the ionospheric tail region itself. For this reason, the downstream electric field exhibits a pronounced cavity, its shape coinciding with the region of reduced solar wind density at the obstacle's nightside. As will be discussed in the following sections, the asymmetry in Titan's ionospheric tail structure is based on exactly the same mechanism. 

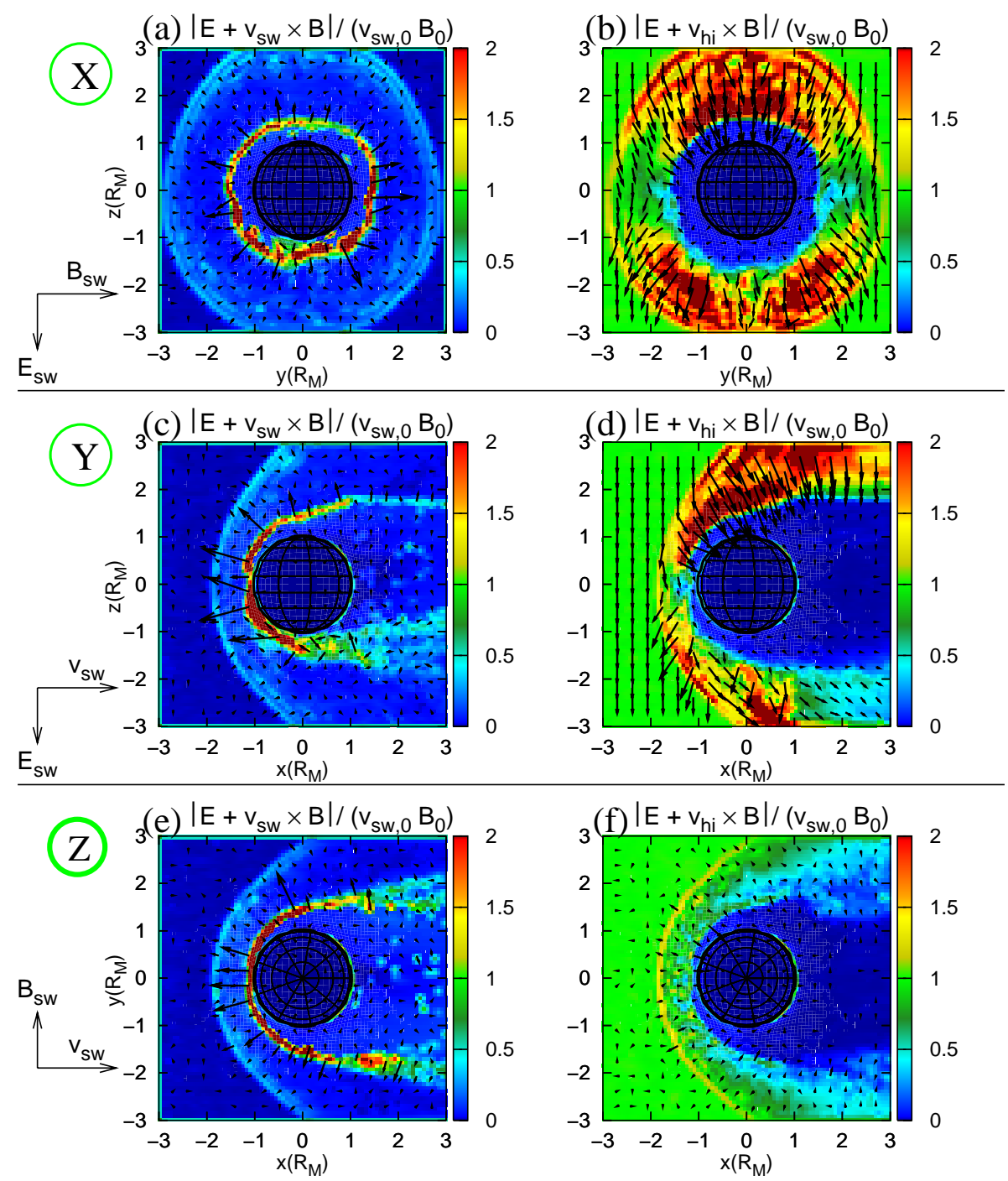

Fig. 3. Plasma environment of Mars - Lorentz forces. The figure diplays the Lorentz forces acting on solar wind ( $S W)$ and ionospheric heavy ions ( $H I)$ for the terminator plane: (a) and (b), the polar plane: (c) and (d), and the equatorial plane: (e) and (f). The arrows denote the projection of the force vectors on the cutting planes. The Lorentz force is given in units of $v_{S W, 0} B_{0}$, i.e. the quantity displayed in the figure is dimensionless. The cutting planes $(x=0),(y=0)$ and $(z 0)$ are denoted by the abbreviations X, Y and Z, respectively. Mach numbers of the upstream plasma flow: $M_{A}=10.0$ (alfvénic), $M_{S}=5.1$ (sonic) and $M_{M S}=4.5$ (magnetosonic).

In an analogeous manner, one can discuss the dynamics of a solar wind proton in the northern hemisphere of the polar plane, attempting to cross the Ion Composition Boundary from outward to inward. When trying to get into the tail region, the proton velocity $\boldsymbol{v}_{S W}$ is approximately the ion bulk velocity $\boldsymbol{u}_{S W}$, so that the convective electric field in the Lorentz force equation cancels with the $\boldsymbol{v}_{S W} \times \boldsymbol{B}$ term. Consequently, the force acting on the particle is clearly dominated by the two electron pressure terms. As the solar wind density inside the tail is almost zero, the major force acting on the proton arises from the ionospheric density gradient near the northern flank of the tail:

$$
\begin{aligned}
\boldsymbol{F}_{S W} & =-e \frac{\nabla\left(n_{e, S W}\right)^{\kappa}+\nabla\left(n_{e, H I}\right)^{\kappa}}{e\left(n_{e, S W}+n_{e, H I}\right)} \\
& \approx-2 \nabla n_{e, H I} .
\end{aligned}
$$

When entering the tail region from outward to inward, the ionospheric particle density increases, i.e. the density gradient vector is directed perpendicular to the tail's flank and points inward. Thus, the Lorentz force on the solar wind particle, as described by Eq. (17), is directed perpendicular to the tail and points outward. This force is the major reason for the solar wind particles to be forbidden to enter the tail region from outward to inward. 
Inside the ionospheric tail itself, the solar wind particles are only of minor importance. As the mean heavy ion velocity $\boldsymbol{u}_{H I}$ inside the tail region does not differ significantly from the individual particle velocities $\boldsymbol{v}_{H I}$ and the magnetic field is weak, the forces acting on the heavy ions inside the tail region are mainly governed by local density gradients, i.e.

$$
\boldsymbol{F}_{H I} \approx-2 e \frac{\nabla n_{e, H I}}{e}
$$

Since according to Fig. 2 b no strong density gradients occur in the central tail region beyond the obstacle, the characteristic time scale for the motion of the heavy ions is very large.

The kinetic approach discussed above also allows to explain the lack of an ICB in the southern hemisphere. In this hemispere, the convective electric field is directed away from the obstacle. Therefore, it drags the ionospheric particles away from Mars, giving rise to an extended pick-up region (cf. Fig. 2e). At the tail's outer flank in this hemisphere, both the electric field arising from the electron density gradient and the convective electric field are directed away from Mars, i.e. the vectors are antiparallel to the z-axis. Due to the anti-parallelism of both forces being the essential condition for the formation of the Ion Composition Boundary, such a boundary layer is not formed in the southern hemisphere.

The highly asymmetric structure of the ionospheric tail and the ICB surrounding it is not only confirmed by other simulation studies (Bößwetter et al., 2004; Modolo et al., 2005), but also by observations. The existence of an asymmetry is suggested by Vennerstrom et al. (2003) who conducted a statistical analysis of the magnetic field data collected by MGS. The same phenomenon is discussed by Brain et al. (2005, 2006). However, a detailed global data analysis concerning the formation of the ICB has not yet been performed. Due to permanent changes in the direction of the solar wind magnetic field and therefore in the direction of the convective electric field, carrying out such an analysis is extremely difficult. Therefore, even though the decisive role of the electric field direction has already been emphasized by numerical models, including this aspect into data analysis will be left to future work.

The qualitative discussion that has been given above is in complete agreement to the results of our simulation model. In order to demonstrate this, the Lorentz forces acting on solar wind and ionospheric heavy ions are displayed in Fig. 3, not only for the polar plane, but also for cuts through the terminator plane and the equatorial plane. As shown in Figs. 3a, $c$ and $e$, in the vicinity of Mars the Lorentz forces acting on solar wind protons are always directed away from the obstacle. A sudden increase of Lorentz force strength manifests in all three cutting planes, denoting the position of the Ion Composition Boundary. It is obvious that the deflection of the ionospheric particles away from Mars occurs primarily in these regions. As can be seen in Figs. 3b and d, in the northern hemisphere where the undisturbed convective

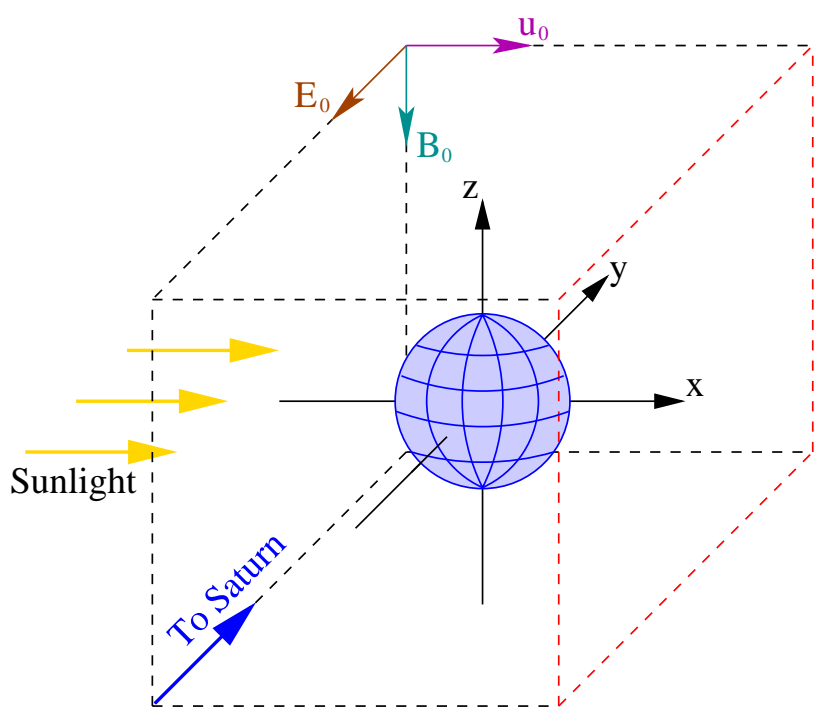

Fig. 4. Plasma environment of Titan - Simulation geometry. The dayside of the obstacle is located in the $(x<0)$ hemisphere. The undisturbed plasma flow is directed parallel to the $(+x)$-axis, whereas the undisturbed Saturnian magnetic field is perpendicular to Titan's orbital plane, pointing in $(-\mathrm{z})$-direction. When Titan is located inside Saturn's magnetosphere, the y-axis is pointing towards Saturn. The boundary layer denoted by the red lines is located in Titan's wake region. In this geometry, the $(x, y)$ plane of the coordinate system coincides with Titan's equatorial plane, whereas the polar plane is identical to the $(x, z)$ plane. This situation differs from the Martian simulation geometry displayed in Fig. 1.

electric field is pointing in the direction of the $(-z)$-axis, the Lorentz force acting on particles of ionospheric origin is always oriented towards Mars. This yields a sharp confinement of the ionospheric particles to the near-obstacle region. In the southern hemisphere, the convective electric field is directed away from the obstacle. Correspondingly, the Lorentz forces acting on ionospheric particles are pointing away from Mars in the southern hemisphere of the terminator plane, as displayed in Fig. 3b. Since the Lorentz forces acting on the two different ion species are parallel in this hemisphere, an Ion Composition Boundary cannot be formed. As displayed in Fig. 3d, an analogeus process occurs in the southern hemisphere of the polar plane. However, it is also obvious that in this hemisphere, the Lorentz force on ionospheric oxygen ions possesses a component parallel to the direction of the undisturbed solar wind flow, cleary illustrating the pick-up of these particles.

Finally, it should be noticed that the second term in Eq. (2) is of no consequence for the discussion of the asymmetries in the wake region of the polar plane. The numerator of this term can be expressed as

$$
(\nabla \times \boldsymbol{B}) \times \boldsymbol{B}=-\frac{1}{2} \nabla \boldsymbol{B}^{2}+(\boldsymbol{B} \cdot \nabla) \boldsymbol{B},
$$



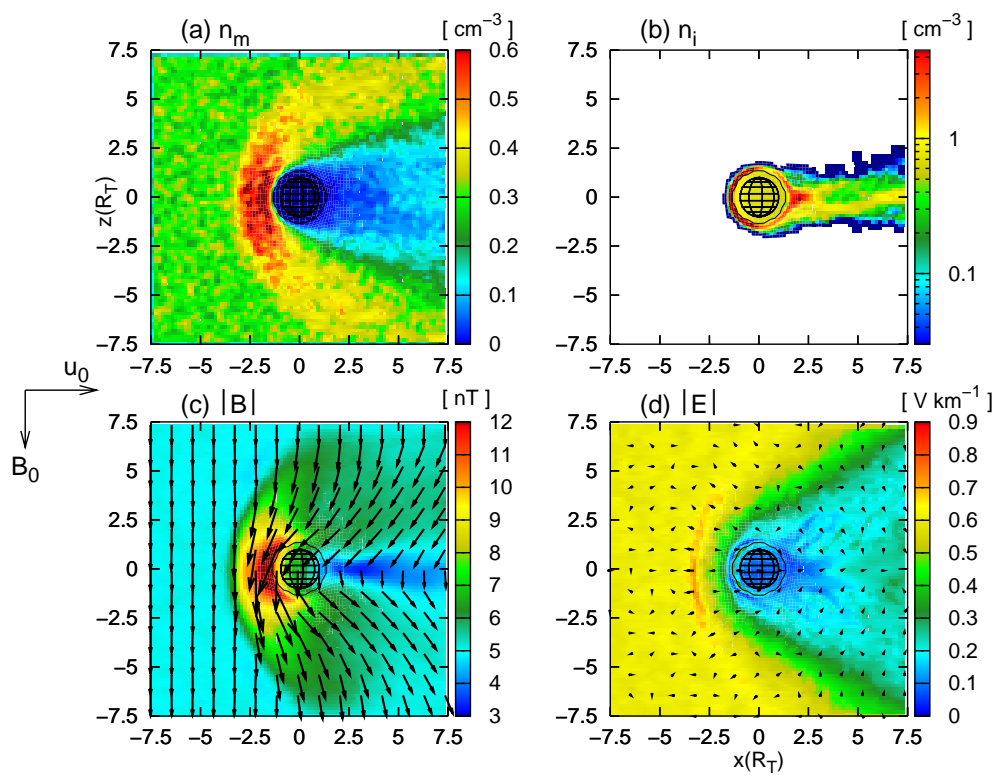

Fig. 5. Titan's interaction with a slightly super-alfvénic, supersonic and supermagnetosonic plasma flow - Cut through the polar plane. The figure displays (a) the ambient plasma density, (b) the ionospheric plasma density and the electromagnetic fields: (c) and (d). Mach numbers of the upstream plasma flow: $M_{A}=1.87$ (alfvénic), $M_{S}=2.9$ (sonic) and $M_{M S}=1.6$ (magnetosonic).
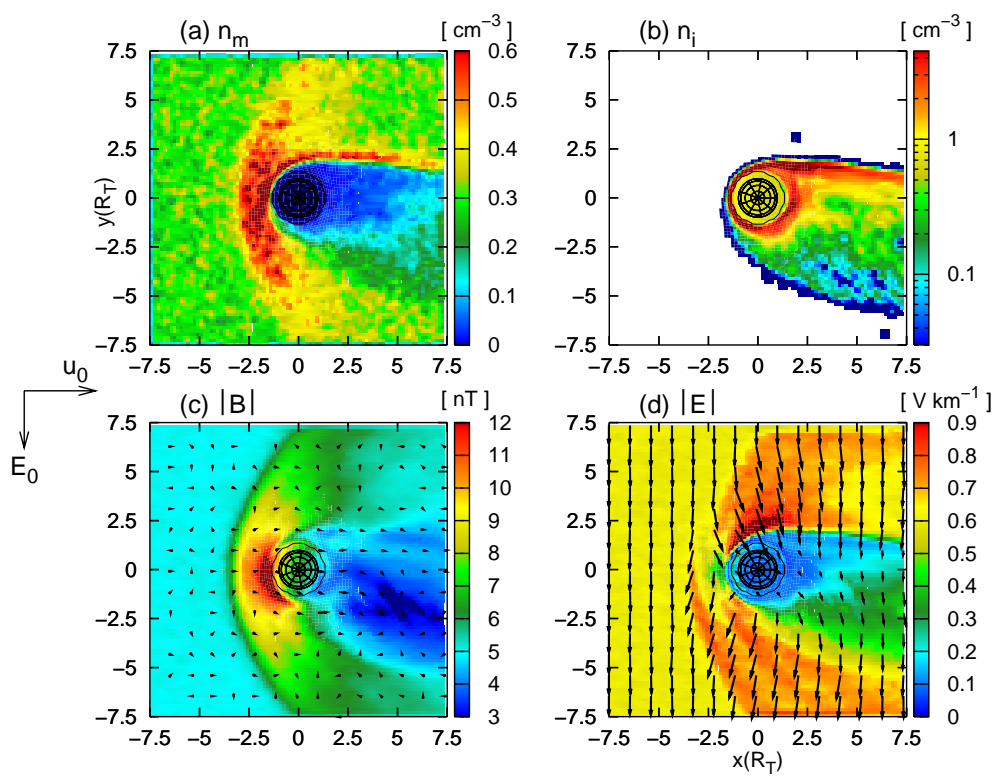

Fig. 6. Titan's interaction with slightly super-alfvénic, supersonic and supermagnetosonic plasma flow - Cut through the equatorial plane. The physical quantities shown in the figure are the same as in Fig. 5. Mach numbers of the upstream plasma flow: $M_{A}=1.87$ (alfvénic), $M_{S}=2.9$ (sonic) and $M_{M S}=1.6$ (magnetosonic).

showing that it is determined by the magnetic pressure gradient as well as the magnetic tension in regions of curved magnetic field lines. The magnetic pressure term plays an important role for the structure of the magnetic pile-up region at the Martian ramside. Besides, magnetic pressure effects have a decisive character for the structure of the magnetic lobes. However, neither of these two forces is important for the ionospheric tail structure in the polar plane, representing a cut through the neutral region between the two lobes. As can be seen from Fig. 2c, the magnetic field strength in the downstream region either vanishes or shows only a very slight increase. For this reason, the discussion presented above does not consider the second term in Eq. (2). For the case of weak comets, an analogeous approach has been 

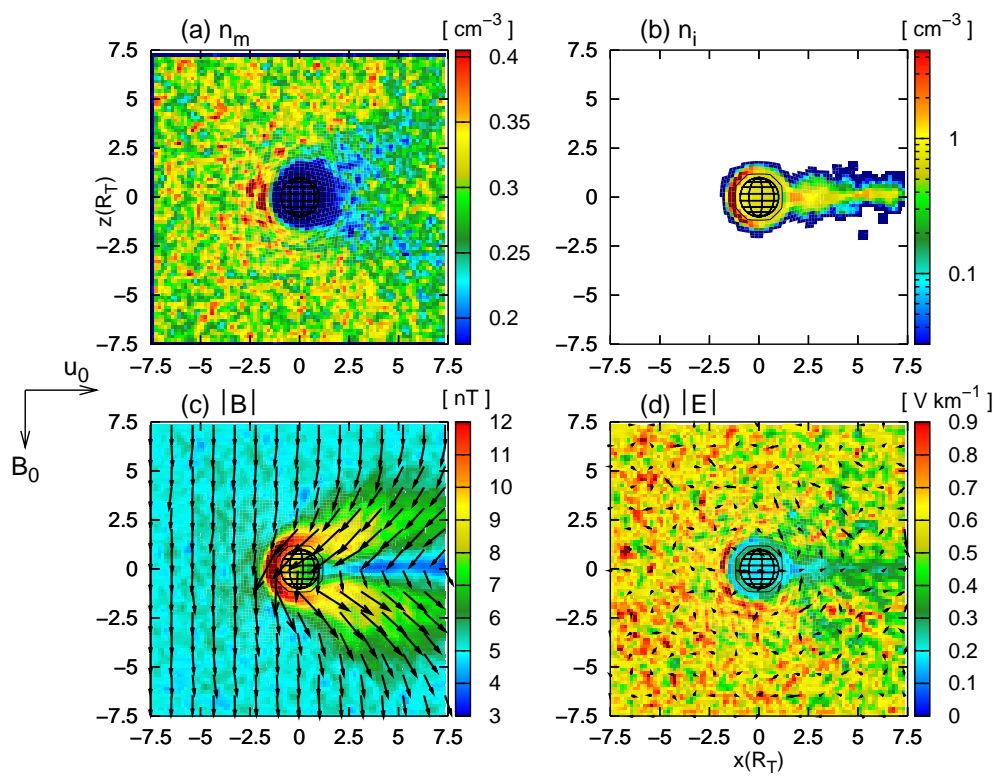

Fig. 7. Interaction between Titan's ionosphere and a slightly super-alfvénic, yet subsonic and submagnetosonic plasma flow - Cut through the polar plane. The physical quantities shown in the figure are the same as in Fig. 5. Mach numbers of the upstream plasma flow: $M_{A}=1.87$ (alfvénic), $M_{S}=0.57$ (sonic) and $M_{M S}=0.55$ (magnetosonic).
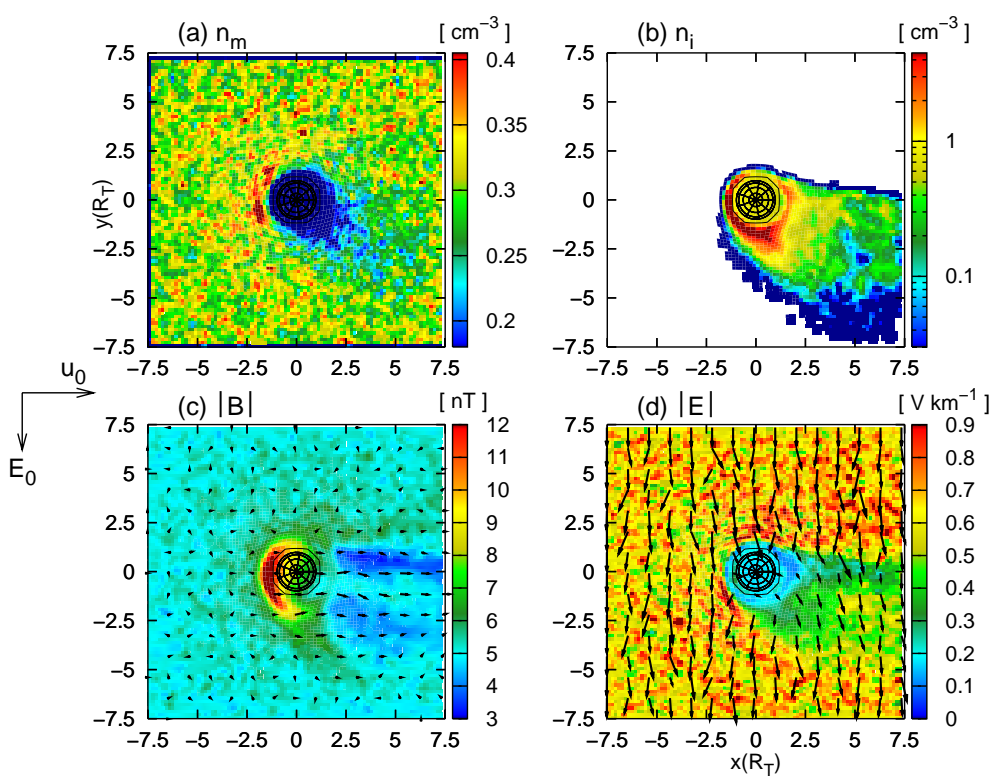

Fig. 8. Interaction between Titan's ionosphere and a slightly super-alfvénic, yet subsonic and submagnetosonic plasma flow - Cut through the equatorial plane. The physical quantities shown in the figure are the same as in Fig. 5. Mach numbers of the upstream plasma flow: $M_{A}=1.87$ (alfvénic), $M_{S}=0.57$ (sonic) and $M_{M S}=0.55$ (magnetosonic).

chosen by Bagdonat (2004). The importance of magnetic effects for the ionospheric tail structure in the equatorial plane, containing the highly curved field lines, has been discussed by Bößwetter et al. (2004).

\section{Titan in the solar wind $\left(M_{A}>1, M_{S}>1, M_{M S}>1\right)$}

When Saturn's magnetosphere is compressed due to high solar wind dynamic pressure, the magnetosphere in the subsolar region can move beyond Titan's orbit and hence, Titan can interact directly with the solar wind. In the following section we are interested in the transition that Titan's 
plasma environment undergoes when the satellite re-enters the magnetosphere. Therefore, four different simulation scenarios have been considered. At first, the case of Titan being exposed to a slightly super-alfvénic $\left(M_{A}=1.87\right)$, supersonic $\left(M_{S}=2.9\right)$ and supermagnetosonic $\left(M_{M S}=1.6\right)$ plasma flow has been analyzed. Even though the Mach numbers are significantly smaller and closer to 1 than in the Martian scenario, the global features of the interaction region in both cases should exhibit many similarities. Secondly, the interaction between Titan's ionosphere and the plasma in Saturn's outer magnetosphere has been analyzed. In this scenario, the upstream flow is super-alfvénic, yet subsonic and submagnetosonic.

A third geometry which is characterized by a superalfvénic and supersonic, yet submagnetosonic flow, illustrates the transition between the situation outside and inside the magnetosphere. A fourth geometry refers to the case of Titan being located in Saturn's magnetotail.

In order to allow a direct comparison between the results, the upstream plasma flow is assumed to be consisting of a single ion species of mass $m=9.6$ amu and density $n_{m}=0.3 \mathrm{~cm}^{-3}$ in all four simulations. These parameters are average values for a plasma consisting of nitrogen $\left(\mathrm{N}^{+}\right)$and hydrogen $\left(\mathrm{H}^{+}\right)$ions and can be obtained from the Voyager 1 data (Kallio et al., 2004; Backes et al., 2005; Simon et al., 2006b). In the following, this plasma is referred to as the $\left(\mathrm{N}^{+} / \mathrm{H}^{+}\right)$plasma.

The simulation geometry is displayed in Fig. 4. The (+x)axis is pointing from the Sun to Titan, whereas the undisturbed background magnetic field $\boldsymbol{B}_{0}=(0,0,-5 \mathrm{nT})$ is oriented antiparallel to the z-axis. The undisturbed plasma velocity $\boldsymbol{u}_{0}=(120 \mathrm{~km} / \mathrm{s}, 0,0)$ is directed parallel to the $\mathrm{x}$-axis. Of course, these parameters do not represent real solar wind conditions and have been chosen to achieve the same characteristic plasma scales for the situation inside and outside the magnetosphere. This supersonic scenario has simply been generated from the case of Titan being located inside the magnetosphere by reducing the plasma temperature, all other upstream parameters are the same. Especially the plasma composition does not represent real solar wind conditions. However, as this case will only provide a qualitative reference to understand Titan's interaction with Saturn's magnetospheric plasma, it is only of minor quantitative interest. A similar approach to identify the global characteristics of Titan's interaction with a supersonic flow has been chosen by Kabin et al. (1999, 2000); Kallio et al. (2004) and Simon et al. (2006b).

For the case of all three Mach numbers being larger than 1 , a plot of the ambient $\left(n_{m}\right)$ and ionospheric $\left(n_{i}\right)$ ion densities as well as the electromagnetic field quantities is given in Figs. 5 and 6, respectively.

The major features of the interaction region are quite similar to the Martian plasma environment. A sharply pronounced bow wave arises in front of the obstacle, denoting a sudden decrease of plasma velocity. In the plane perpen- dicular to the convective electric field, the structure of the interaction region is highly symmetric (cf. Fig. 5), whereas an asymmetry with respect to the direction of the convective electric field is formed in the equatorial plane. In the polar plane, a cone-shaped wake region is formed downstream of the obstacle, denoting a significant decrease of the ambient plasma density. In strong analogy, the ionospheric particle density is high inside the cone-shaped wake region, whereas it vanishes outside the tail. The mechanism giving rise to the asymmetries in the structure of the Ion Composition Boundary is well illustrated by the cut through the equatorial plane (cf. Fig. 6). In the $(y>0)$ hemisphere, where the convective electric field points towards Titan, a sharp increase of ionospheric plasma density manifests near the outer flank of the ionospheric tail. This gives rise to a field $\boldsymbol{E} \propto-\nabla P_{e}$. Therefore, the $\left(\mathrm{N}^{+} / \mathrm{H}^{+}\right)$ions which are dragged against this barrier from outside by the convective electric field are unable to pass this region, yielding a line-like region of increased $\left(\mathrm{N}^{+} / \mathrm{H}^{+}\right)$density near the outer flank of the boundary layer.

\section{Titan in Saturn's outer magnetosphere $\left(M_{A}>1\right.$, $\left.M_{S}<1, M_{M S}<1\right)$}

The set of Mach numbers for the case of Titan being located in Saturn's outer magnetosphere has been chosen in accordance to the data collected during the Voyager 1 flyby of Titan (Ness et al., 1982; Backes, 2005): the alfvénic Mach number is given by $M_{A}=1.87$, whereas values of $M_{S}=0.57$ and $M_{M S}=0.55$ have been chosen for the sonic and the magnetosonic Mach numbers, respectively.

When Titan is located in the outer regions of Saturn's magnetosphere, the impinging plasma flow is super-alfvénic, yet subsonic and submagnetosonic. Consequently, no bow shock will form in front of the obstacle. The simulation results for this scenario are displayed in Fig. 7 for the polar plane and in Fig. 8 for the equatorial plane. As can be seen in Fig. 8b, the structure of the ionospheric tail is still highly asymmetric with respect to the direction of the convective electric field, but however, the magnetospheric plasma $\left(n_{m}\right)$ is no longer separated from the ionospheric plasma flow, i.e. the Ion Composition Boundary has vanished.

As displayed in Fig. 8, at the tail's outer flank in the $(y>0)$ hemisphere, the convective electric field is still directed perpendicular to the tail and pointing inward. Therefore the tail is forbidden to expand into this hemisphere. This is the reason why in both the case of supersonic and in the case of subsonic upstream flow the structure of the tail exhibits an asymmetry with respect to the direction of the convective electric field. In the plane perpendicular to $E$, the situation is highly symmetric. For the case of Titan being located inside Saturn's magnetosphere, the existence of such an asymmetry has been confirmed during the first Cassini flybys, as discussed in an analysis of cold plasma measurements by Wahlund et al. (2005). 

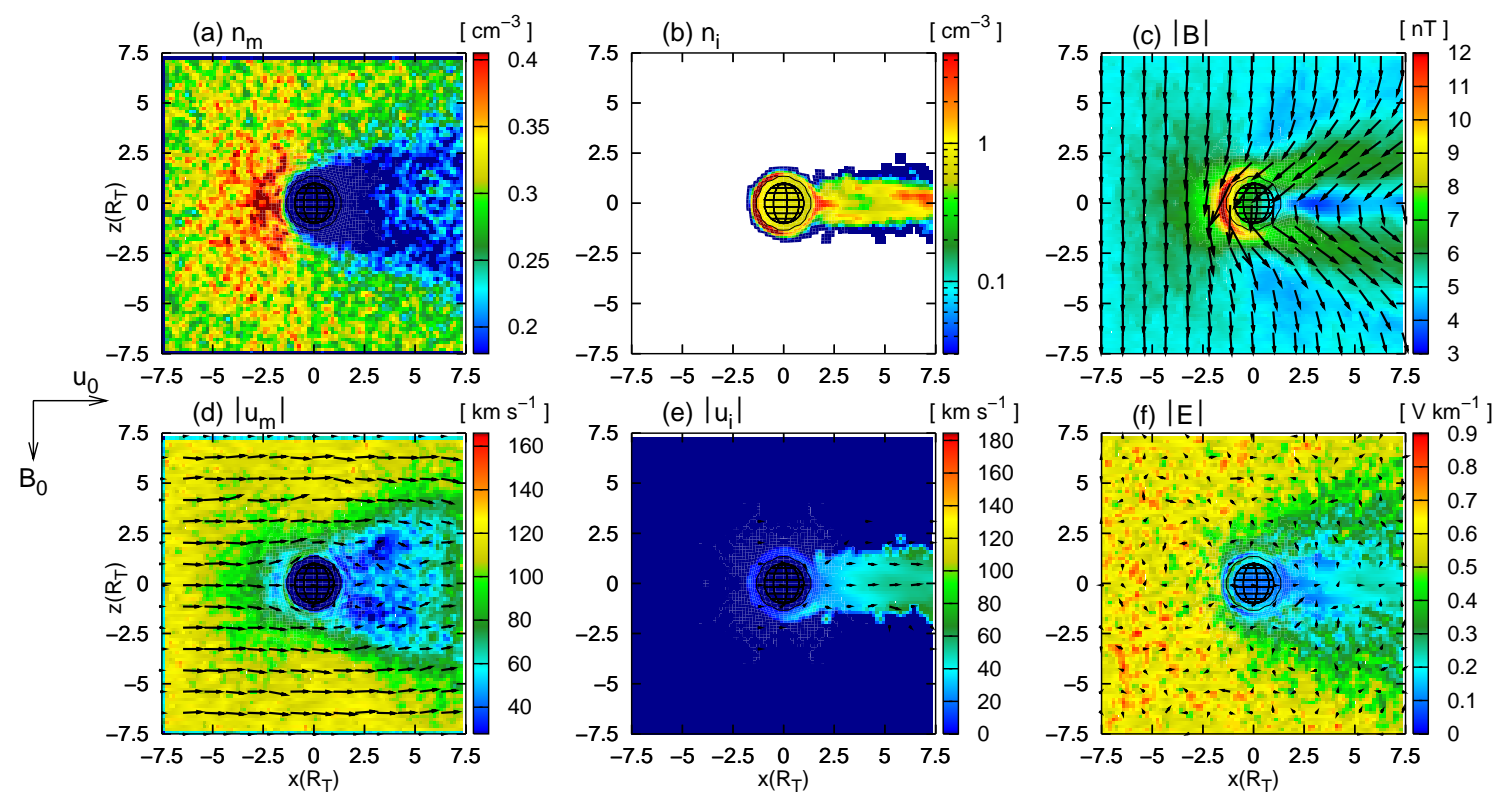

Fig. 9. Transition from supermagnetosonic to submagnetosonic flow - Cut through the $(x, z)$ plane of the coordinate system which coincides with Titan's polar plane. The figure displays the $\left(\mathrm{N}^{+} / \mathrm{H}^{+}\right)$plasma density and velocity: (a) and (d), the ionospheric ion density and velocity: (b) und (e), and the electromagnetic fields: (c) and (f). The figure illustrates the transition from the case of Titan being located outside the magnetosphere, where all three Mach numbers are larger than 1, to the case of Titan's interaction with Saturn's magnetospheric plasma at 18:00 LT. Mach numbers of the upstream plasma flow: $M_{A}=1.87$ (alfvénic), $M_{S}=1.08$ (sonic) and $M_{M S}=0.94$ (magnetosonic).

However, as can be seen in Fig. 8b, only a slight decrease of ionospheric plasma density occurs near the outer flank of the ionospheric tail. Due to the adiabatic description of the electron fluid, the electric field arising from the pressure gradient is too weak to prevent the magnetospheric plasma from mixing with the ionospheric plasma flow. For this reason, only a minor, but nevertheless still asymmetric decrease of magnetospheric plasma density occurs in the vicinity of the obstacle. The Ion Composition Boundary has completely vanished. In contrast to this, the situation in the $(y<0)$ hemisphere is very similar to the case of Titan being loacted outside of the magnetosphere. The electric field is directed away from the obstacle, leading to the formation of an extended pick-up region. A more extensive discussion for this scenario is given by Simon et al. (2006b). In the following section, we intend to focus on illustrating the physical process that results in the disappearence of the Ion Composition Boundary.

\section{Transition from supersonic to subsonic flow $\left(M_{A}>1, M_{S}>1, M_{M S}<1\right)$}

In this section, the transition between the two scenarios described in the preceding sections shall be investigated in more detail. For this reason, another simulation run has been conducted. In order to gain access to the transition between the cases of Titan being located outside and inside the magnetosphere, the upstream plasma flow is assumed to be super-alfvénic $\left(M_{A}>1\right)$ and supersonic $\left(M_{S}>1\right)$, yet sub- magnetosonic $\left(M_{M S}<1\right)$. Specifically, values of $M_{A}=1.87$, $M_{S}=1.08$ and $M_{M S}=0.94$ have been chosen for the Mach numbers of the impinging flow, corresponding to values of $\beta_{e, m}=0.97$ and $\beta_{m}=2.00$ for the $\left(\mathrm{N}^{+} / \mathrm{H}^{+}\right)$plasma's electron and ion betas, respectively. All other simulation parameters, including the upstream plasma composition as well as the background magnetic field and density are the same as in the other Titan simulation runs presented in this work. Even though this situation is not representative for a real situation in Titan's plasma environment, it will show to be extremely valuable for unterstanding the transition that the obstacle's plasma environment undergoes.

The simulation geometry is displayed in Fig. 4, i.e. the situation refers again to the 18:00 local time geometry at the equinoxes of Saturn's orbital period. The simulation results are shown in Figs. 9 and 10, respectively. It is obvious that the pronounced shock front has vanished, but nevertheless, a cone-shaped region of reduced plasma density is still identifiable in the downstream region. Figure 9 displays the characteristic plasma parameters and the electromagnetic fields for a cut through the polar plane, coinciding with the $(x, z)$ plane of the coordinate frame. It should be noticed that since the magnetosonic Mach number is now smaller than 1 , the fast mode velocity in the plasma can become larger than the plasma flow velocity itself. As shown in Fig. 9a, the clearly developed shock front at the obstacle's dayside has vanished. Nevertheless, a diffuse region of enhanced $\left(\mathrm{N}^{+} / \mathrm{H}^{+}\right)$plasma density is still present, denoting an increase 

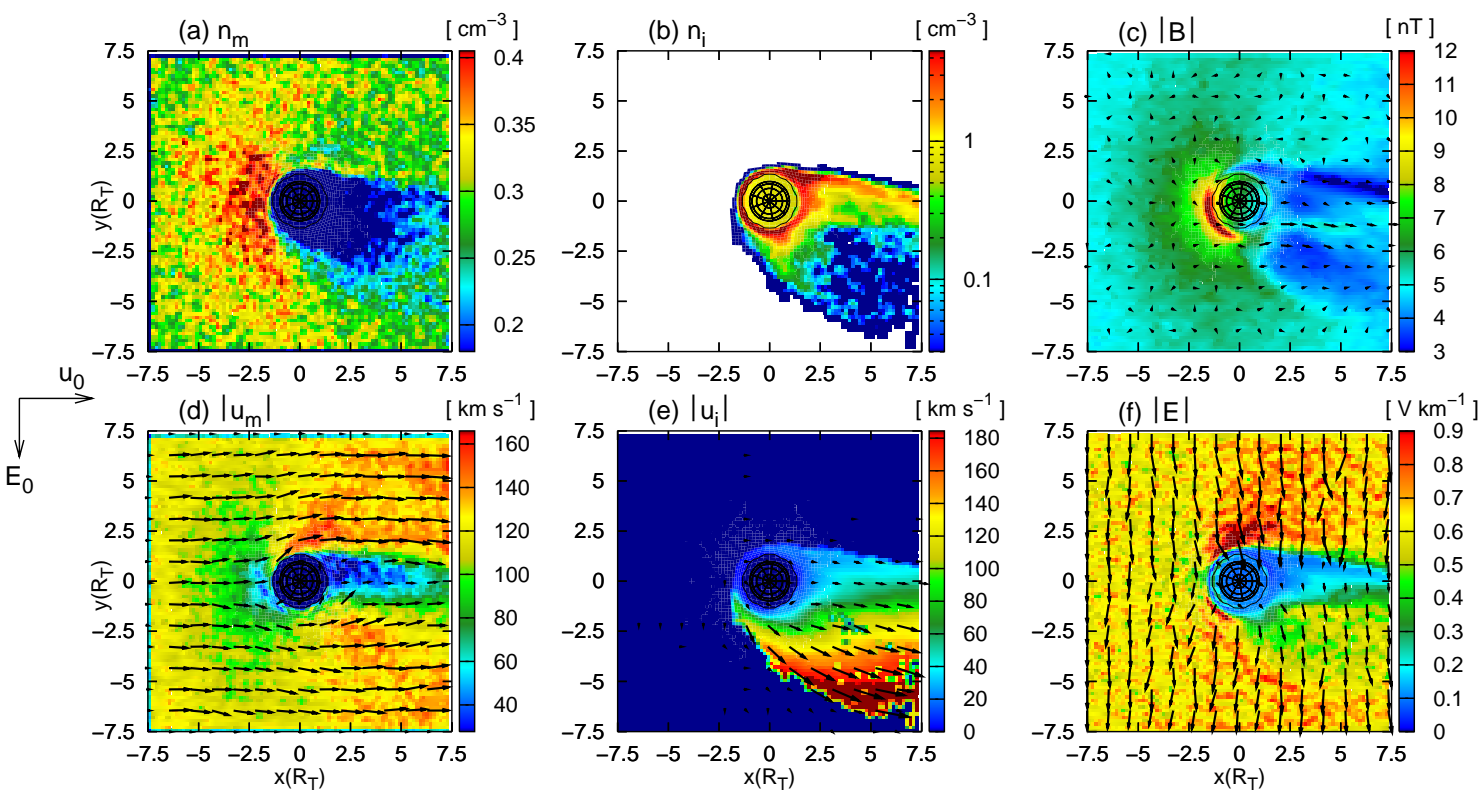

Fig. 10. Transition from supermagnetosonic to submagnetosonic flow - Cut through Titan's equatorial plane which is parallel to the undisturbed convective electric field. The physical quantities shown in the figure are the same as in Fig. 9. Mach numbers of the upstream plasma flow: $M_{A}=1.87$ (alfvénic), $M_{S}=1.08$ (sonic) and $M_{M S}=0.94$ (magnetosonic).

from $n=0.3 \mathrm{~cm}^{-3}$ in the undisturbed flow to $n=0.4 \mathrm{~cm}^{-3}$ in the near-Titan upstream region. However, this structure is not as sharply confined as in the case of Titan being located outside of the magnetosphere.

Besides, a pronounced region of reduced plasma velocity in the downstream region has shown to be characteristic for the interaction region when Titan is exposed to the solar wind, as discussed by Simon et al. (2006b). Such a decrease is still present in the case currently under consideration, as can be seen from Fig. 9d. Although the plasma is still decelerated, the respective region in the polar plane is not sharply separated from the ambient plasma flow. In the case of Titan being located outside the magnetosphere, the interaction in the polar plane gave rise to a cone-shaped region of reduced $\left(\mathrm{N}^{+} / \mathrm{H}^{+}\right)$density in the vicinity of Titan, its outer boundaries denoting the position of the Ion Composition Boundary in a plane perpendicular to the undisturbed convective electric field $\boldsymbol{E}_{0}=-\boldsymbol{u}_{0} \times \boldsymbol{B}_{0}$. This is displayed in Fig. 5a. An analogeous cone-like structure can be seen in Fig. 9a, although the decrease of $\left(\mathrm{N}^{+} / \mathrm{H}^{+}\right)$density has significantly diminished. Besides, the structure denotes no longer a sharp, but a smooth transition from the plasma inside the cone-shaped region to the ambient plasma flow.

The transition that the tail structure undergoes is also illustrated in Fig. 11. Plot 11a displays a cut through the tail structure of the supermagnetosonic case, whereas a cut through the tail in the submagnetosonic case is shown in Fig. 11b. Both cutting planes are located at $x=+5 R_{T}$ downstream of Titan. As can be seen in Fig. 11a, in the supermagnetosonic case, the density cavity in the wake exhibits a sharply pro- nounced boundary along the $y=1 R_{T}$ line, i.e. the density strongly decreases on a characteristic scale below one Titan radius. In contrast to this, when moving away from the obstacle in negative y-direction, a quite smooth density increase occurs, illustrating that no sharp boundary layer exists in the $(y<0)$ hemisphere. As can be seen from Fig. 11b, in the submagnetosonic case, the structure of the density cavity is still highly asymmetric. However, a smooth transition to the ambient density does not only occur in the $(y<0)$, but also in the $(y>0)$ hemisphere. The ambient density in the $\left(x=+5 R_{T}\right)$ cutting plane is smaller than in the supermagnetosonic case, as the shock denoing an increase of plasma density has vanished. To sum up, the position of the former Ion Composition Boundary is still identifiable, but the impinging plasma is no longer forbidden to cross this boundary layer and to mix with the ionospheric pick-up ions. A possible reason for this might be the increased plasma temperature, allowing a larger number of particles with high thermal velocity to cross the potential barrier at the tail's flank. These signatures clearly illustrate the transition to the case of Titan being located inside Saturn's magnetosphere, where the boundary layer has completely vanished.

The transition is also illustrated by the magnetic field signature in the polar plane, as displayed in Fig. $9 \mathrm{c}$. In the case of Titan being located outside the magnetosphere, $B$ exhibits a curved shock structure (cf. Fig. $5 \mathrm{c}$ and $6 \mathrm{c}$ ), whereas when Titan is located inside the magnetosphere, the situation in the polar plane is dominated by a pronounced magnetic draping pattern, being confinded to a narrow region with a diameter of $\pm 4 R_{T}$ perpendicular to the undisturbed flow direction 

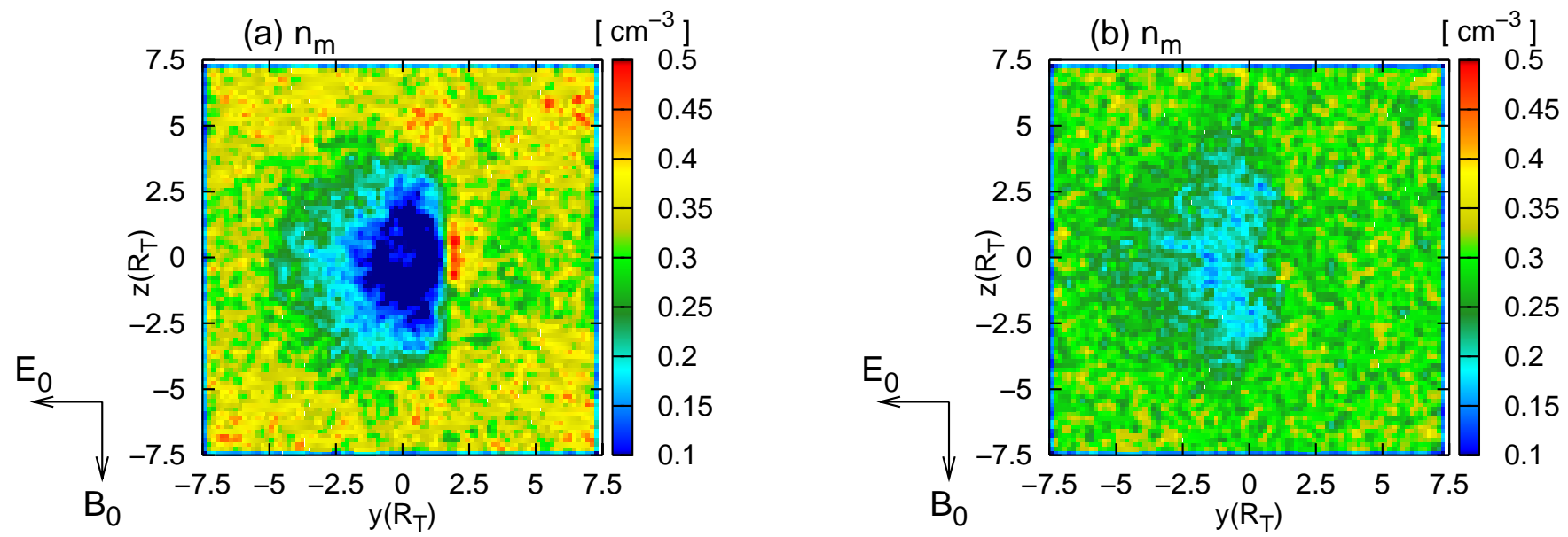

Fig. 11. Transition from supersonic to subsonic flow. The figure illustrates the transition that the tail structure downstream of the obstacle undergoes when the magnetosonic Mach number of the upstream plasma flow is reduced from $M_{M S}>1$ to $M_{M S}<1$. (a) displays a cut through the tail in the supermagnetosonic case at a distance of $5 R_{T}$ to the center of the obstacle, while an analogeous cut for the submagnetosonic case is shown in (b). The cutting planes are parallel to the $(y, z)$ plane of the coordinate system, i.e. they are located at $x=+5 R_{T}$. Both figures display the ambient plasma density $n_{m}$.

(cf. Fig. 7c). Besides, when Titan is located inside the magnetosphere, the magnetic pile-up region at Titan's dayside possesses an extension of only about $1 R_{T}$ in subsolar direction. In contrast to this, when Titan is located outside the magnetosphere, the magnetic pile-up in the shock front possesses an extension around $3 R_{T}$ in subsolar direction, as displayed in Figs. $5 \mathrm{c}$ and $6 \mathrm{c}$. The transition between both cases is illustrated in Fig. 9c. On the one hand, the interaction leads to the formation of a confined magnetic draping pattern, being similar to the situation when Titan is located inside the magnetosphere at 18:00 LT. On the other hand, a slight increase of magnetic field strength can be noticed at a subsolar distance of around $2-3 R_{T}$, denoting the position of the bow shock in the solar wind scenario. Besides, the magnetic enhancement in the two lobes achieves a maximum value of about $7 \mathrm{nT}$, whereas a value of $9-10 \mathrm{nT}$ is reached when Titan is located in the magnetosphere at 18:00 LT. This implies that in the transition scenario under consideration, the field lines are incapable of draping completely around the obstacle, but develop an intermediate structure between a parabolic, barely confined shock front and a strongly confined draping pattern. To summarize the major results for the polar plane, the boundary structures being typical for the interaction region when Titan is located outside the magnetosphere are still identifiable in the transition scenario. However, the sharpness of the boundaries, especially the Ion Composition Boundary, has clearly diminished.

Nevertheless, the most important aspect of the transition scenario is the transformation that the Ion Composition Boundary undergoes in the plane parallel to the undisturbed convective electric field $\boldsymbol{E}_{0}$. The situation in this plane, being highly asymmetric, is displayed in Fig. 10. In the case of Ti- tan being located outside the magnetosphere, the interaction gives rise to a sharply developed Ion Composition Boundary in the $(y>0)$ hemisphere. As can be seen in Fig. 6b, a sharp increase of ionospheric density manifests near the tail's flank in the $(y>0)$ hemisphere. The ambient $\left(\mathrm{N}^{+} / \mathrm{H}^{+}\right)$ plasma is pressed against the outer flank of the ionospheric tail by the convective electric field, but however, due to the electric field arising from the electron pressure gradient, it is incapable of crossing the boundary from outward to inward. This effect yields a region of increased $\left(\mathrm{N}^{+} / \mathrm{H}^{+}\right)$plasma density along the flank of the boundary layer. In the case of Titan being located inside the magnetosphere, the region of sharply increased ionospheric density in the $(y>0)$ hemisphere has vanished, as shown in Fig. 8b. Therefore, the two plasma populations are now allowed to mix. The density plots in Figs. 10a and $\mathrm{b}$ illustrate the transition between both cases: As the decrease of ionospheric density in the $(y>0)$ hemisphere is weaker than in the situation displayed in Fig. 6b, the ionospheric electron pressure gradient has diminished. This is why the ambient $\left(\mathrm{N}^{+} / \mathrm{H}^{+}\right)$plasma is capable of gaining access to the ionospheric tail region. Nevertheless, the former position of the boundary is still identifiable.

\section{Titan in Saturn's magnetotail $\left(M_{A}<1, \quad M_{S}<1\right.$, $\left.M_{M S}<1\right)$}

When Titan is located in Saturn's magnetotail region, the satellite faces a sub-alfvénic, subsonic and submagnetosonic upstream plasma flow (Wolf and Neubauer, 1982; Ledvina et al., 2004). For the situation at the equinoxes of Saturn's orbital period, i.e. when Titan is shielded from the solar UV radiation by Saturn, this scenario has been investigated by 

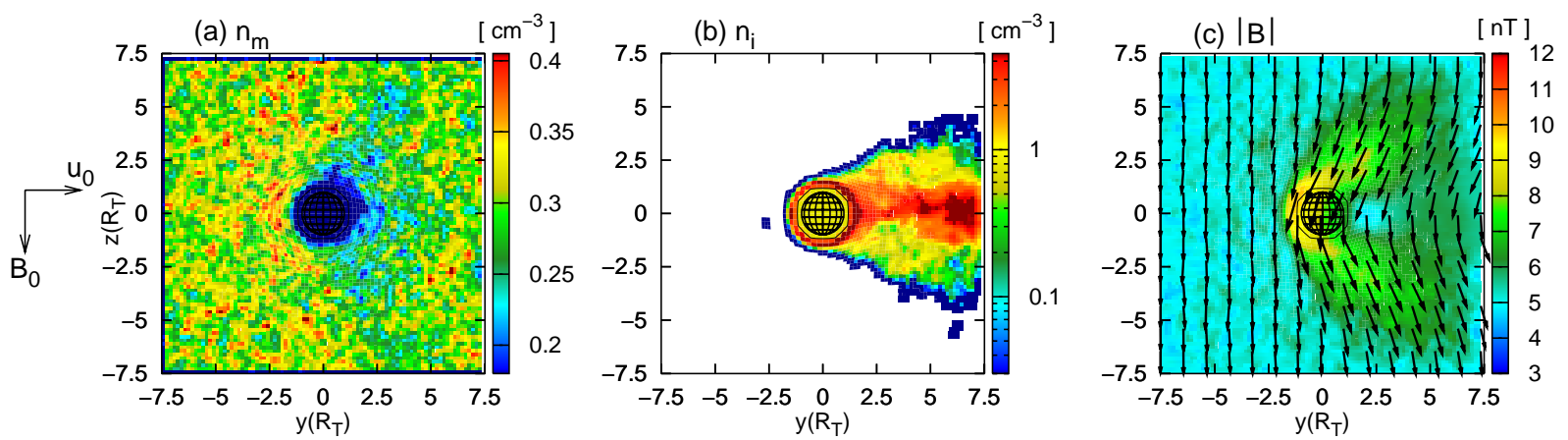

Fig. 12. Titan's interaction with the sub-alfvénic, subsonic and submagnetosonic plasma flow in Saturn's magnetotail region - Cut through the polar plane. The figure displays (a) the magnetospheric plasma density, (b) the ionospheric plasma density, and (c) the magnetic field. Mach numbers of the upstream plasma flow: $M_{A}=0.77$ (alfvénic), $M_{S}=0.27$ (sonic) and $M_{M S}=0.22$ (magnetosonic).
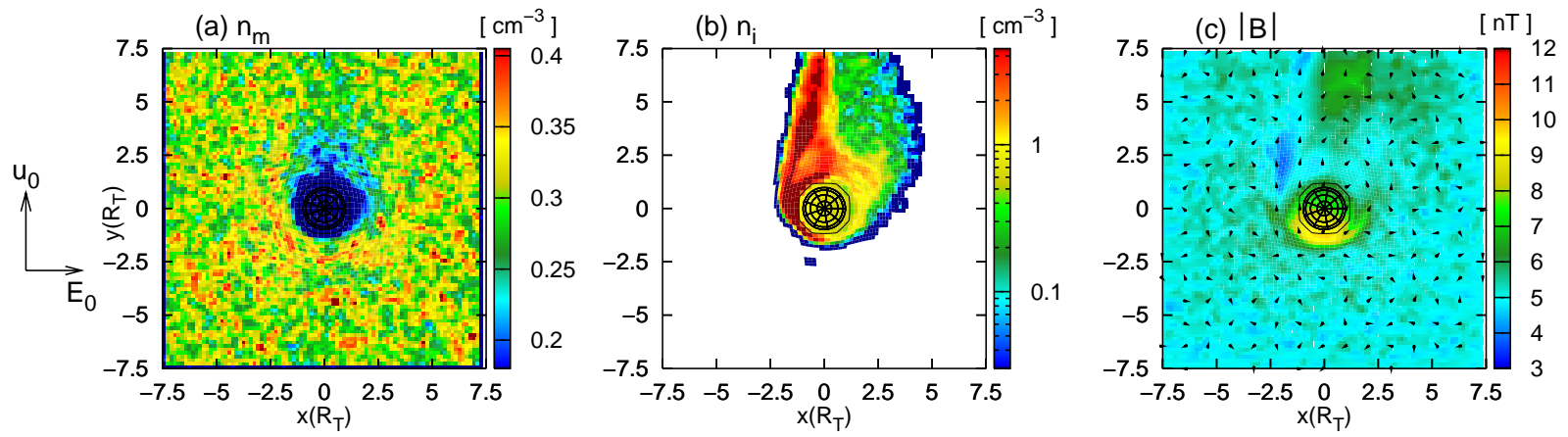

Fig. 13. Titan's interaction with the sub-alfvénic, subsonic and submagnetosonic plasma flow in Saturn's magnetotail region - Cut through the equatorial plane. The figure displays (a) the magnetospheric plasma density, (b) the ionospheric plasma density, and (c) the magnetic field. Mach numbers of the upstream plasma flow: $M_{A}=0.77$ (alfvénic), $M_{S}=0.27$ (sonic) and $M_{M S}=0.22$ (magnetosonic).

Simon et al. (2006b). However, as the Cassini flybys take place in late southern summer, a scenario where the obstacle is not completely protected from solar UV ionization might be more realistic. For this reason, the $(x<0)$ hemisphere of Titan is still assumed to be exposed to solar UV radiation. Of course, the geometry of the Cassini flybys is significantly more complex, i.e. Titan's orbital plane is not parallel to the Sun-Titan-line. Therefore, the real situation of Titan being located inside Saturn's magnetotail during the Cassini flybys may probably be located between the case discussed here and the scenario of a completely shielded obstacle, as suggested by Simon et al. (2006b). Here, our primary purpose is to keep the simulation geometry as simple as possible to get a good insight into the relevant plasma processes.

The $\mathrm{x}$-axis is again directed from the Sun to Titan, whereas the undisturbed magnetic field is oriented in $(-\mathrm{z})$-direction. However, in this scenario, the undisturbed plasma flow velocity is parallel to the y-axis. Even though this geometry does not represent the real complexity of the situation in late southern summer, it may be considered a closer approximation to the real situation than the scenario discussed by Simon et al. (2006b). Besides, by retaining the original ionospheric Chapman profile, it is evident that any changes in the struc- ture of the interaction region will occur due to the altered Mach numbers of the upstream flow. For the simulation, the Mach numbers in the undisturbed plasma have been set to $M_{A}=0.77, M_{S}=0.29$ and $M_{M S}=0.27$. The results for the polar plane are displayed in Fig. 12, whereas Fig. 13 shows the results for Titan's equatorial plane.

As can be seen in Figs. 12c and 13c, both the magnetic pile-up region at Titan's ramside and the magnetic lobes are significantly less pronounced than in the other cases under consideration. In the polar plane, a pronounced region of increased ionospheric plasma density arises between the two lobes. In order to stabilize the tail structure, the magnetic pressure in the two lobes must balance the thermal plasma pressure in the region of slightly reduced field strength between the lobes, directly beyond the obstacle. This yields an enhanced ionospheric plasma density in the wake region, as displayed in Fig. 12b. In this scenario, the diameter of the ionospheric tail in the polar plane is significantly larger than in the other cases under consideration. However, neither in the polar plane nor in the equatorial plane, the magnetospheric plasma is separated from the ionospheric plasma flow. As can be seen in Fig. 13b, the ionospheric plasma tail is still drawn in the $(x>0)$ hemisphere by the convective 
electric field, but the outer boundary of the ionospheric tail does not manifest in the magnetospheric plasma density (cf. Fig. 13a). Even though a region of enhanced ionospheric plasma density is formed near the outer flank of the tail, the density gradient in this region (i.e. the decrease of ionospheric plasma density per unit length) is not as sharp as in the case of Titan's interaction with a supersonic, superalfvénic and supermagnetosonic flow (cf. Figs. $6 \mathrm{~b}$ and 13b). However, the maximum density value that is reached near the outer flank of the tail is almost the same in both scenarios. The absolute density value is not of major importance for the effects occuring at the flank of the tail.

\section{Summary}

In this paper, the plasma environments of Mars and Titan have been studied by means of global, 3-D hybrid simulations. Emphasis has been placed on the physical mechanism leading to the formation of a so-called Ion Composition Boundary, separating the ionospheric plasma population from the ambient plasma flow. The physical effects giving rise to such a structure have been investigated as a function of the alfvénic, sonic and magnetosonic Mach numbers of the upstream plasma flow. In the following, the major results will be summarized by discussing the transition that the obstacle's plasma environment undergoes when the values of the three Mach numbers are reduced from very high values (plasma environment of Mars) to very low values (Titan in Saturn's magnetotail).

In the Martian situation, the ionospheric plasma flow is clearly separated from the solar wind by a sharphy developed boundary layer which is reproduced by simulations as well as by spacecraft measurements. This boundary layer is highly symmetric in a plane perpendicular to the convective electric field, whereas it exhibits a pronounced asymmetry with respect to the direction of this field. The underlying physical mechanism has been explained in terms of a kinetic model, considering the Lorentz forces that act on ions of solar wind and ionospheric origin. The major element of this intepretation is a combination of convective electric field and electron pressure forces, leading to the formation of a sharply pronounced boundary layer in the hemisphere where both forces are antiparallel. In the hemisphere where the two forces are parallel, the ionospheric particles are dragged away from the obstacle, leading to a significant extension of the ionospheric tail. These considerations are valid for the Martian plasma environment as well as for the case of Titan being exposed to a slightly super-alfvénic, supersonic and supermagnetosonic plasma flow.

When Titan is exposed to the plasma flow in Saturn's outer magnetosphere, the ionospheric tail is still asymmetric with respect to the direction of the electric field. However, the boundary layer between both plasma populations has almost vanished because the electron pressure gradient has signif- icantly diminished. For this reason, it is unable to prevent both plasma populations from mixing. The transition between Titan's plasma environment outside and inside Saturn's magnetosphere has been illustrated by means of a third scenario, assuming the upstream flow to be super-alfvénic, supersonic and submagnetosonic. In this geometry, the former position of the Ion Composition Boundary is still identifiable, although its sharpness has clearly diminished and both plasmas start mixing. The simulation results clearly illustrate that the slower is the upstream flow, the less pronounced is the separation of ionospheric and magnetospheric plasma flow. This tendency also manifests in the results for Titan being located in Saturn's magnetotail, where the upstream flow is sub-alfvénic, subsonic and submagnetosonic.

To sum up, based on our simulation results, it seems that only in a Mach number regime of $1 \leq M_{A}$ a more or less sharply pronounced Ion Composition Boundary is formed due to the interaction of an unmagnetized planet's ionosphere with its plasma environment. If the alfvénic Mach number is smaller, the influence of the convective electric field, causing the asymmetry in the tail structure, does not undergo a significant change. However, as the sharpness of the ionospheric tail's outer boundary clearly diminishes, the electric force ramp arising form the $-\nabla P_{e}$ term is unable to forbid the ambient flow to enter the tail region.

On the one hand, our future work will focus on analyzing the early Martian plasma environment, especially to obtain a comparison to the present situation. Besides, it will be interesting to analyze how the position and structure of the ICB are affected by the strong crustal anomalies at Mars. Harnett and Winglee $(2003,2005)$ who studied the influence of these anomalies by using a non-ideal MDH model suggest that these anomalies do not only cause a local modification of the boundary layers, but cause a strong distorsion of the ionospheric tail structure on a characteristic length scale of several planetary radii. Since the influence of the magnetic anomalies has not yet been considered by the hybrid approach, such a modification of the existing simulation model will be realized in the near future.

On the other hand, the analysis of Titan's plasma environment shall be continued, the simulation geometry matching the situation during specific Cassini flybys. An extension of the model to multispecies upstream conditions will be a first step in this direction.

Acknowledgements. This work has been supported by the Deutsche Forschungsgemeinschaft through the grants MO 539/13-1 and MO 539/ 15-1. Besides, the authors thank J. Schuele from the Institute for Scientific Computing, TU Braunschweig, for numerous valuable discussions concerning the parallelization of the 3-D simulation code.

Topical Editor I. A. Daglis thanks a referee for her/his help in evaluating this paper. 


\section{References}

Acuña, M. H., Connerney, J. E. P., Wasilewski, P., Lin, R. P., Anderson, K. A., Calson, C. W., McFadden, J., Curtis, D. W., Mitchell, D., Rème, H., Mazelle, C., Sauvaud, J. A., d'Uston, C., Cros, A., Medale, J. L., Bauer, S. J., Cloutier, P., Mayhew, M., Winterhalter, D., and Ness, N. F.: Magnetic field and plasma observations at Mars: Initial results of the Mars Global Surveyor Mission, Science, 279, 1676-1680, 1998.

Backes, H.: Titan's Interaction with the Saturnian Magnetospheric Plasma, Ph.D. thesis, Universität Köln, 2005.

Backes, H., Neubauer, F. M., Dougherty, M. K., Achilleos, N., André, N., Arridge, C. S., Bertucci, C., Jones, G. H., Khurana, K. K., Russell, C. T., and Wennmacher, A.: Titan's Magnetic Field Signature During the First Cassini Encounter, Science, 308, 992-995, 2005.

Bagdonat, T.: Hybrid Simulation of Weak Comets, Ph.D. thesis, Technische Universität Braunschweig, 2004.

Bagdonat, T. and Motschmann, U.: 3D hybrid simulation of solar wind interaction with comets, in: Space Plasma Simulation Proceedings of the Sixth International School/ Symposium ISSS6, edited by: Büchner, J., Dum, C., and Scholer, M., 80-83, 2001.

Bagdonat, T. and Motschmann, U.: 3D Hybrid Simulation Code Using Curvilinear Coordinates, J. of Computational Physics, 183, 470-485, 2002a.

Bagdonat, T. and Motschmann, U.: From a weak to a strong comet - 3D global hybrid simulation studies, Earth, Moon and Planets, 90, 305-321, 2002b.

Barabash, S. and Lundin, R.: ASPERA-3 on Mars Express, Icarus, 182, 301-307, 2006.

Bertucci, C., Mazelle, C., Crider, D. H., Mitchell, D. L., Sauer, K., Acuña, M. H., Connerney, J. E. P., Lin, R. P., Ness, N. F., and Winterhalter, D.: MGS MAG/ER observations at the magnetic pileup boundary of Mars: draping enhancement and low frequency waves, Adv. Space Res., 33, 1938-1944, 2004.

Bertucci, C., Mazelle, C., and Acuña, M. H.: Interaction of the solar wind with Mars from Mars Global Surveyor MAG/ER observations, J. Atmos. T. Phys., 67, 1797-1808, 2005a.

Bertucci, C., Mazelle, C., Acuña, M. H., Russell, C. T., and Slavin, J. A.: Structure of the magnetic pileup boundary at Mars and Venus, J. Geophys. Res., 110, 1209-1217, 2005b.

Bößwetter, A., Bagdonat, T., Motschmann, U., and Sauer, K.: Plasma boundaries at Mars: A 3D simulation study, Ann. Geophys., 22, 4363-4379, 2004, http://www.ann-geophys.net/22/4363/2004/.

Brain, D. A., Halekas, J. S., Lillis, R., Mitchell, D. L., Lin, R. P., and Crider, D. H.: Variability of the altitude of the Martian sheath, Geophys. Res. Lett., 32, L18203, doi:10.1029/2005GL023126, 2005.

Brain, D. A., Mitchell, D. L., and Halekas, J. S.: The magnetic field draping direction at Mars from April 1999 through August 2004, Icarus, 182, 464-473, 2006.

Brecht, S., Luhmann, J. G., and Larson, D. J.: Simulation of the Saturnian magnetospheric interaction with Titan, J.Geophys. Res., 105, 13 119-13 130, 2000.

Breus, T. K., Krymskii, A. M., Lundin, R., Dubinin, E. M., Luhmann, J. G., Yeroshenko, Y. G., Barabash, S. V., Mitnitskii, V. Y., Pissarenko, N. F., and Styashkin, V. A.: The solar wind interaction with Mars: consideration of Phobos-2 mission observations of an ion composition boundary on the dayside, J.Geophys. Res., 96, $11165-11174,1991$.

Harnett, E. M. and Winglee, R. M.: The influence of a minimagnetopause on the magnetic pileup boundary at Mars, Geophys. Res. Lett., 30, 10-1, 2003.

Harnett, E. M. and Winglee, R. M.: Three-dimensional fluid simulations of plasma asymmetries in the Martian magnetotail caused by the magnetic anomalies, J. Geophys. Res., 110, 7226-7238, 2005.

Kabin, K., Gombosi, T. I., DeZeeuw, D. L., Powell, K. G., and Israelevich, P. L.: Interaction of the Saturnian magnetosphere with Titan: Results of a three-dimensional MHD simulation, J.Geophys. Res., 104, 2451-2458, 1999.

Kabin, K., Israelevich, P. L., Ershkovich, A. I., Neubauer, F. M., Gombosi, T. I., DeZeeuw, D. L., and Powell, K. G.: Titan's magnetic wake: Atmospheric or magnetospheric interaction, J.Geophys. Res., 105, 10 761-10770, 2000.

Kallio, E. and Janhunen, P.: Ion escape from Mars in a quasi-neutral hybrid model, J. Geophys. Res., 107, 1-1, 2002.

Kallio, E., Sillanpää, I., and Janhunen, P.: Titan in subsonic and supersonic flow, Geophys. Res. Lett., 31, L15 703/1-L15 703/4, 2004.

Ledvina, S. A. and Cravens, T. E.: A three-dimensional MHD model of plasma flow around Titan, Planet. Space Sci., 46, 11751191, 1998.

Ledvina, S. A., Luhmann, J. G., Brecht, S. H., and Cravens, T. E.: Titan's induced magnetosphere, Advances in Space Research, 33, 2092-2102, 2004.

Luhmann, J. G., Russell, C. T., Schwingenschuh, K., and Yeroshenko, Y.: A comparison of induced magnetotails of planetary bodies: Venus, Mars and Titan, J.Geophys. Res., 96, 11 199-11 208, 1991.

Lundin, R., Barabash, S., Andersson, H., Holmström, M., Grigoriev, A., Yamauchi, M., Sauvaud, J.-A., Fedorov, A., Budnik, E., Thocaven, J.-J., Winningham, D., Frahm, R., Scherrer, J., Sharber, J., Asamura, K., Hayakawa, H., Coates, A., Linder, D. R., Curtis, C., Hsieh, K. C., Sandel, B. R., Grande, M., Carter, M., Reading, D. H., Koskinen, H., Kallio, E., Riihela, P., Schmidt, W., Säles, T., Kozyra, J., Krupp, N., Woch, J., Luhmann, J., McKenna-Lawler, S., Cerulli-Irelli, R., Orsini, S., Maggi, M., Mura, A., Milillo, A., Roelof, E., Williams, D., Livi, S., Brandt, P., Wurz, P., and Bochsler, P.: Solar Wind-Induced Atmospheric Erosion at Mars: First Results from ASPERA-3 on Mars Express, Science, 305, 1933-1936, 2004.

Mazelle, C., Rème, H., Sauvaud, J. A., d'Uston, C., Carlson, C. W., Anderson, K. A., Curtis, D. W., Lin, R. P., Korth, A., Mendis, D. A., Neubauer, F. M., Glassmeier, K. H., and Raeder, J.: Analysis of suprathermal electron properties at the magnetic pile-up boundary of comet P/Halley, 16, 1035-1038, 1989.

Modolo, R., Chanteur, G. M., Dubinin, E., and Matthews, A. P.: Influence of the solar EUV flux on the Martian plasma environment, Ann. Geophys., 23, 433-444, 2005, http://www.ann-geophys.net/23/433/2005/.

Nagy, A. F., Winterhalter, D., Sauer, K., Cravens, T. E., Brecht, S., Mazelle, C., Crider, D., Kallio, E., Zakharov, A., Dubinin, E., Verigin, M., Kotova, G., Axford, W. I., Bertucci, C., and Trotignon, J. G.: The plasma Environment of Mars, Space Science Reviews, 111, 33-114, 2004.

Ness, N., Acuna, M., Behannon, K., and Neubauer, F.: The induced 
magnetosphere of Titan, J. Geophys. Res., 87, 1369-1381, 1982.

Neubauer, F. M., Gurnett, D. A., Scudder, J. D., and Hartle, R. E.: Titan's magnetospheric interaction, in: Saturn, edited by: Gehrels, T. and Matthews, M. S., Univ. Arizona Press, Tucson, 760-787, 1984.

Rème, H., Mazelle, C., Sauvaud, J. A., d'Uston, C., Froment, F., Lin, R. P., Anderson, K. A., Carlson, C. W., Larson, D. E., Korth, A., Chaizy, P., and Mendis, D. A.: Electron Plasma Environment at Comet Grigg-Skjellerup: General Observations and Comparison With the Environment at Comet Halley, J. Geophys. Res., 98, 20 965-20 976, 1993.

Riedler, W., Schwingenschuh, K., Lichtenegger, H., Möhlmann, D., Rustenbach, J., Weroshenko, Y., Achache, J., Slavin, J., Luhmann, J. G., and Russell, C. T.: Interaction of the solar wind with the planet Mars: Phobos-2 magnetic field observations, Planet. Space Sci., 39, 75-81, 1991.

Sauer, K., Roatsch, T., Motschmann, U., Moehlmann, D., and Schwingenschuh, K.: Plasma boundaries at Mars discovered by the PHOBOS 2 magnetometers, Ann. Geophys., 8, 661-670, 1990 ,

http://www.ann-geophys.net/8/661/1990/.

Sauer, K., Bogdanov, A., and Baumgärtel, K.: Evidence of an ion composition boundary (protonopause) in bi-ion fluid simulations of solar wind mass loading, Geophys. Res. Lett., 21, 2255-2258, 1994.

Schardt, A. W., Behannon, K. W., Lepping, R. P., Carbary, J. F., Eviatar, A., and Siscoe, G. L.: The outer magnetosphere, in: Saturn, edited by: Gehrels, T. and Matthews, M. S., University of Arizona Press, Tucson, 416-459, 1984.

Shimazu, H.: Three-dimensional hybrid simulation of solar wind interaction with unmagnetized planets, J. Geophys. Res., 106, 8333-8342, 2001.

Simon, S., Bagdonat, T., Motschmann, U., and Glassmeier, K.-H.: Plasma environment of magnetized asteroids: A 3-D hybrid simulation study, Ann. Geophys., 24, 407-414, 2006 a.
Simon, S., Boesswetter, A., Bagdonat, T., Motschmann, U., and Glassmeier, K.-H.: Plasma environment of Titan: A 3-D hybrid simulation study, Ann. Geophys., 24, 1113-1135, 2006b.

Terada, N., Machida, S., and Shinagawa, H.: Global hybrid simulation of the Kelvin-Helmholtz instability at the Venus ionopause, J. Geophys. Res., 107, 30-1, 2002.

Vennerstrom, S., Olsen, N., Purucker, M., Acuña, M. H., and Cain, J. C.: The magnetic field in the pile-up region at Mars, and its variation with the solar wind, Geophys. Res. Lett., 30, 22-1, 2003.

Verigin, M. I., Gringauz, K. I., and Ness, N. F.: Comparison of induced magnetospheres at Venus and Titan, J. Geophys. Res., 89, 5461-5470, 1984.

Vignes, D., Mazelle, C., Reme, H., Acuña, M. H., Connerney, J. E. P., Lin, R. P., Mitchell, D. L., Cloutier, P., Crider, D. H., and Ness, N. F.: The Solar Wind interction with Mars: locations and shapes of the Bow Shock and the observations of the MAG/ER experiment onboard Mars Global Surveyor, Geophys. Res. Lett., 27, 49-52, 2000.

Wahlund, J.-E., Boström, R., Gustafsson, G., Gurnett, D. A., Kurth, W. S., Pedersen, A., Averkamp, T. F., Hospodarsky, G. B., Persoon, A. M., Canu, P., Neubauer, F. M., Dougherty, M. K., Eriksson, A. I., Morooka, M. W., Gill, R., André, M., Eliasson, L., and Mueller-Wodarg, I.: Cassini Measurements of Cold Plasma in the Ionosphere of Titan, Science, 308, 986-989, 2005.

Wolf, D. A. and Neubauer, F. M.: Titan's Highly Variable Plasma Environment, J. Geophys. Res., 87, 881-885, 1982.

Yeroshenko, Y., Riedler, W., Schwingenschuh, K., Luhmann, J. G., Ong, M., and Russell, C. T.: The Magnetotail of Mars: Phobos Observations, Geophys. Res. Lett., 17, 885-888, 1990. 